\title{
Polyhedral Approaches to Mixed Integer Linear Programming
}

\author{
Michele Conforti \\ Università di Padova, conforti@math.unipd.it \\ Gérard Cornuéjols * \\ Carnegie Mellon University and Université d'Aix-Marseille \\ gc0v@andrew.cmu.edu \\ Giacomo Zambelli \\ Università di Padova, giacomo@math.unipd.it
}

April 2008, revised November 2008

\begin{abstract}
This survey presents tools from polyhedral theory that are used in integer programming. It applies them to the study of valid inequalities for mixed integer linear sets, such as Gomory's mixed integer cuts.
\end{abstract}

Key words: mixed integer linear program, Farkas' lemma, Minkowski-Weyl theorem, polyhedron, union of polyhedra, cutting plane, split cut, Gomory mixed integer cut, Chvátal rank

\section{Introduction}

\subsection{Mixed integer linear programming}

In this tutorial we consider mixed integer linear programs. These are problems of the form

\footnotetext{
* Supported by NSF grant CMMI0653419, ONR grant N00014-03-1-0188 and ANR grant ANR06-BLAN-0375.
} 


$$
\begin{aligned}
\max c x+h y & \\
A x+G y & \leq b \\
x & \geq 0 \text { integral } \\
y & \geq 0,
\end{aligned}
$$

where the data are the row vectors $c \in \mathbb{Q}^{n}, h \in \mathbb{Q}^{p}$, the matrices $A \in \mathbb{Q}^{m \times n}$, $G \in \mathbb{Q}^{m \times p}$ and the column vector $b \in \mathbb{Q}^{m}$; and the variables are the column vectors $x \in \mathbb{R}^{n}$ and $y \in \mathbb{R}^{p}$. The set $S$ of feasible solutions to (1) is called a mixed integer linear set when $p \geq 1$ and a pure integer linear set when $p=0$.

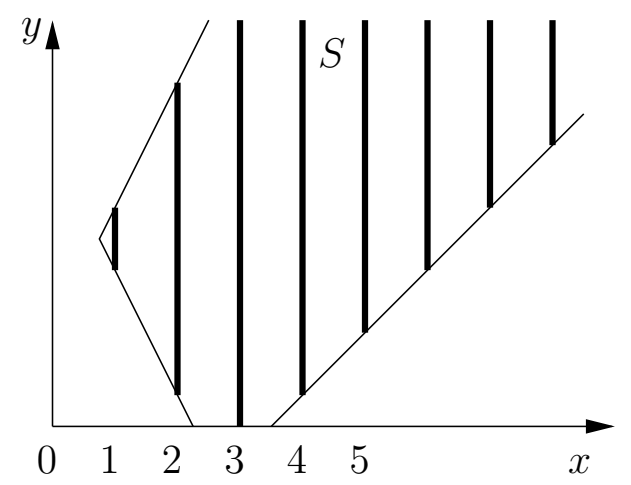

Figure 1: A mixed integer set

The polyhedral approach is a powerful tool for solving mixed integer linear programs (1). This is the topic of this tutorial.

\subsection{Historical perspective}

Babylonian tablets show that mathematicians were already solving systems of linear equations over 3000 years ago. The eighth book of the Chinese Nine Books of Arithmetic, written over 2000 years ago, describes the Gaussian elimination method. In 1809, Gauss [29] used this method in his work and presented it as a "standard technique". Surprisingly, the method was subsequently named after him.

A major breakthrough occurred when mathematicians started analyzing systems of linear inequalities. This is a fertile ground for beautiful theories. In 1826 Fourier [28] gave an algorithm for solving such systems by eliminating variables one at a time. Other important contributions are due to Farkas [26] and Minkowski [39]. Systems of linear inequalities define polyhedra and 
it is natural to optimize a linear function over them. This is the topic of linear programming, arguably one of the greatest successes of computational mathematics in the twentieth century. The simplex method, developed by Dantzig [20] in 1951, is currently used to solve large-scale applications in all sorts of human endeavors. It is often desirable to find integer solutions to linear programs. This is the topic of this tutorial. The first algorithm for solving (1) in the pure integer case was discovered in 1958 by Gomory [31].

When considering algorithmic questions, a fundamental issue is the increase in computing time when the size of the problem instance increases. In the 1960s Edmonds [23] was one of the pioneers in stressing the importance of polynomial-time algorithms. These are algorithms whose computing time is bounded by a polynomial function of the instance size. In particular Edmonds [24] pointed out that, by being a bit careful with the intermediate numbers that are generated, the Gaussian elimination method can be turned into a polynomial-time algorithm. The existence of a polynomial-time algorithm for linear programming remained a challenge for many years. This question was resolved positively by Khachiyan [34] in 1979, and later by Karmarkar [33] using a totally different algorithm. Both algorithms were (and still are) very influential, each in its own way. In integer programming, Lenstra [36] found a polynomial-time algorithm when the number of variables is fixed. Although integer programming is NP-hard in general, the polyhedral approach has proved successful in practice. It can be traced back to the work of Dantzig, Fulkerson and Johnson [21]. Research is currently very active in this area. Also very promising are non-polyhedral approximations that can be computed in polynomial-time, such as semidefinite relaxations (Lovász and Schrijver [37], Goemans and Williamson [30]).

In the next section, we motivate the polyhedral approach by presenting a cutting plane method for solving mixed integer linear programs (1).

\subsection{Cutting Plane Methods}

Solving a mixed integer linear program (MILP) such as (1) is NP-hard (Cook [16]). One approach that has been quite successful in practice is based on an idea that is commonly used in computational mathematics: Find a relaxation that is easier to compute and gives a tight approximation. We focus on linear programming $(L P)$ relaxations.

Given a mixed integer linear set $S:=\left\{(x, y) \in \mathbb{Z}_{+}^{n} \times \mathbb{R}_{+}^{p}: A x+G y \leq b\right\}$, a linear programming relaxation of $S$ is a set $P^{\prime}=\left\{(x, y): A^{\prime} x+G^{\prime} y \leq b^{\prime}\right\}$ that contains $S$. Why LP relaxations? Mainly for two reasons: As mentioned already, there are efficient practical algorithms for solving linear programs 
[20], [33]. Second, one can generate a sequence of LP relaxations that provide increasingly tight approximations of the set $S$.

For a mixed integer set $S$, there is a natural $L P$ relaxation:

$$
P_{0}:=\{(x, y): A x+G y \leq b, x \geq 0, y \geq 0\}
$$

which is obtained from the system that defines $S$ by discarding the integrality requirement on the vector $x$.

Let $\left(x^{0}, y^{0}\right)$ be an optimal solution and $z^{0}$ the value of the linear program

$$
\max \left\{c x+h y:(x, y) \in P_{0}\right\}
$$

whose constraint set is the natural linear programming relaxation of $S$. We will assume that we have a linear programming solver at our disposal, thus $\left(x^{0}, y^{0}\right)$ and $z^{0}$ are available to us. Let $z^{*}$ be the optimal value of (1). Since $S \subseteq P_{0}$, it follows that $z^{0} \geq z^{*}$. Furthermore if $x^{0}$ is an integral vector, then $\left(x^{0}, y^{0}\right) \in S, z^{*}=z^{0}$ and the MILP (1) is solved.

A strategy for dealing with an optimal solution $\left(x^{0}, y^{0}\right)$ of $(2)$ that is not in $S$ is to find an inequality $\alpha x+\gamma y \leq \beta$ that is satisfied by every point in $S$ and such that $\alpha x^{0}+\gamma y^{0}>\beta$. The existence of such an inequality is guaranteed when $\left(x^{0}, y^{0}\right)$ is an optimal basic solution of $(2)$, which can be found by standard LP algorithms.

An inequality $\alpha x+\gamma y \leq \beta$ that is satisfied by every point in $S$ is a valid inequality for $S$. If such an inequality is violated by $\left(x^{0}, y^{0}\right)$, it is a cutting plane separating $\left(x^{0}, y^{0}\right)$ from $S$.

Define now

$$
P_{1}=P_{0} \cap\{(x, y): \alpha x+\gamma y \leq \beta\} .
$$

Since $S \subseteq P_{1} \subset P_{0}$, a linear programming relaxation for MILP based on $P_{1}$ is stronger than the natural relaxation based on $P_{0}$ in the sense that the solution $\left(x^{0}, y^{0}\right)$ of the natural LP relaxation does not belong to $P_{1}$. So the optimal value of the LP

$$
\max \left\{c x+h y:(x, y) \in P_{1}\right\}
$$

is at least as good an approximation of the value $z^{*}$ as $z_{0}$. The recursive application of this idea leads to the cutting plane approach:

Starting with $i=0$, repeat:

Recursive Step: Solve the linear program $\max \left\{c x+h y:(x, y) \in P_{i}\right\}$. If the associated optimal basic solution $\left(x^{i}, y^{i}\right)$ belongs to $S$, Stop.

Otherwise solve the following separation problem: 
Find a cutting plane $\alpha x+\gamma y \leq \beta$ separating $\left(x^{i}, y^{i}\right)$ from $S$.

Set $P_{i+1}:=P_{i} \cap\{(x, y): \alpha x+\gamma y \leq \beta\}$ and repeat the recursive step.

If $\left(x^{i}, y^{i}\right)$ is not in $S$, there are infinitely many cutting planes separating $\left(x^{i}, y^{i}\right)$ from $S$. So the separation problem that is recursively solved in the above algorithm has many solutions. There is usually a tradeoff between the running time of a separation procedure and the quality of the cutting planes it produces. We will discuss several possibilities in this survey.

For now, we illustrate the cutting plane approach on a two variable example (see Figure 2):

$$
\begin{aligned}
\max z=11 x_{1}+4.2 x_{2} & \\
-x_{1}+x_{2} & \leq 2 \\
8 x_{1}+2 x_{2} & \leq 17 \\
x_{1}, x_{2} & \geq 0 \text { integer. }
\end{aligned}
$$

We first add slack variables $x_{3}$ and $x_{4}$ to turn the inequality constraints into equalities. The problem becomes:

$$
\begin{aligned}
z-11 x_{1}-4.2 x_{2} & =0 \\
-x_{1}+x_{2}+x_{3} & =2 \\
8 x_{1}+2 x_{2}+x_{4} & =17 \\
x_{1}, x_{2}, x_{3}, x_{4} & \geq 0 \text { integer. }
\end{aligned}
$$

Solving the LP relaxation, we get the optimal tableau:

$$
\begin{aligned}
z+1.16 x_{3}+1.52 x_{4} & =28.16 \\
x_{2}+0.8 x_{3}+0.1 x_{4} & =3.3 \\
x_{1}-0.2 x_{3}+0.1 x_{4} & =1.3 \\
x_{1}, x_{2}, x_{3}, x_{4} & \geq 0
\end{aligned}
$$

The corresponding basic solution is $x_{3}=x_{4}=0, x_{1}=1.3, x_{2}=3.3$ with objective value $z=28.16$. Since the values of $x_{1}$ and $x_{2}$ are not integer, this is not a solution of (3). We can generate a cut from the constraint $x_{2}+0.8 x_{3}+0.1 x_{4}=3.3$ using the following reasoning. Since $x_{2}$ is an integer variable, we have

$$
0.8 x_{3}+0.1 x_{4}=0.3+k \quad \text { where } k \in \mathbb{Z} .
$$

Since the left-hand-side is nonnegative, we must have $k \geq 0$, which implies

$$
0.8 x_{3}+0.1 x_{4} \geq 0.3
$$

This is the famous Gomory fractional cut [31]. Note that it cuts off the above fractional LP solution $x_{3}=x_{4}=0$. 


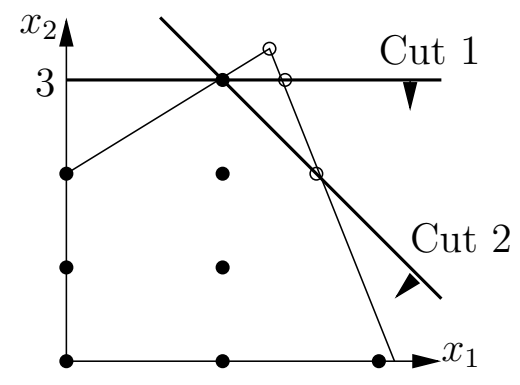

Figure 2: The first two cuts in the cutting plane algorithm

Since $x_{3}=2+x_{1}-x_{2}$ and $x_{4}=17-8 x_{1}-2 x_{2}$, we can express Gomory's fractional cut in the space $\left(x_{1}, x_{2}\right)$. This yields $x_{2} \leq 3$ (see Figure 2).

Adding this cut to the linear programming relaxation, we get the following formulation.

$$
\begin{aligned}
\max & 11 x_{1}+4.2 x_{2} \\
& -x_{1}+x_{2} \leq 2 \\
& 8 x_{1}+2 x_{2} \leq 17 \\
& x_{2} \leq 3 \\
& x_{1}, x_{2} \geq 0
\end{aligned}
$$

Solving this linear program, we find the basic solution $x_{1}=1.375, x_{2}=3$ with value $z=27.725$. From the optimal tableau, we can generate a new fractional cut:

$$
x_{1}+x_{2} \leq 4
$$

Adding this cut to the LP, we find a new LP optimum $x_{1}=1.5, x_{2}=2.5$ with value $z=27$. Two more iterations are needed to obtain the optimal integer solution $x_{1}=1, x_{2}=3$ with value $z=23.6$.

\section{Polyhedra and the Fundamental Theorem of In- teger Programming}

A polyhedron in $\mathbb{R}^{n}$ is a set of the form $P:=\left\{x \in \mathbb{R}^{n}: A x \leq b\right\}$ where $A$ is a real matrix and $b$ a real vector. If $A$ and $b$ have rational entries, $P$ is a rational polyhedron. A polyhedron of the form $\left\{x \in \mathbb{R}^{n}: A x \leq 0\right\}$ is called a polyhedral cone. Note that a polyhedral cone is always nonempty since it contains the null vector 0 . 
For $S \subseteq \mathbb{R}^{n}$, the convex hull of $S$ is the set $\operatorname{conv}(S):=\left\{x \in \mathbb{R}^{n}: x=\right.$ $\sum_{i=1}^{k} \lambda_{i} x^{i}$ where $k \geq 1, \lambda \in \mathbb{R}_{+}^{k}, \sum_{i=1}^{k} \lambda_{i}=1$ and $\left.x^{1}, \ldots, x^{k} \in S\right\}$. This is the smallest convex set that contains $S$. Note that $\operatorname{conv}(\emptyset)=\emptyset$. The convex hull of a finite set of points in $\mathbb{R}^{n}$ is called a polytope. It will sometimes be useful to work with $\overline{\operatorname{conv}}(S)$, the closure of $\operatorname{conv}(S)$, which is the smallest closed convex set that contains $S$. The conic hull of a nonempty set $S \subseteq \mathbb{R}^{n}$ is cone $(S):=\left\{x \in \mathbb{R}^{n}: x=\sum_{i=1}^{k} \lambda_{i} x^{i}\right.$ where $k \geq 1, \lambda \in \mathbb{R}_{+}^{k}$ and $x^{1}, \ldots, x^{k} \in$ $S\}$. If $S$ is a finite set, $\operatorname{cone}(S)$ is said to be finitely generated. It will be convenient to define cone $(\emptyset)=\{0\}$.

Given a cone $C$ and $r \in C \backslash\{0\}$, the set cone $(r)=\{\lambda r: \lambda \geq 0\}$ is called a ray of $C$. Since cone $(\lambda r)=\operatorname{cone}(r)$ for every $\lambda>0$, we will sometimes simply refer to a vector $r \in C$ as a ray, to denote the corresponding ray cone $(r)$. So when we say that $r$ and $r^{\prime}$ are distinct rays, we mean cone $(r) \neq \operatorname{cone}\left(r^{\prime}\right)$. We say that $r \in C \backslash\{0\}$ is an extreme ray if there are no distinct rays $r^{1}$ and $r^{2}$ such that $r=r^{1}+r^{2}$. We say that $C$ is pointed if, for every $r \in C \backslash\{0\}$, $-r \notin C$. One can verify that if $C$ is a finitely generated pointed cone, then $C$ is generated by its extreme rays.

An important theorem, due to Minkowski and Weyl, states that every polyhedron $P$ can be written as the sum of a polytope $Q$ and a finitely generated cone $C$. Here $Q+C:=\left\{x \in \mathbb{R}^{n}: x=y+z\right.$ for some $y \in$ $Q$ and $z \in C\}$. Note that $P=\emptyset$ if and only if $Q=\emptyset$. If $P:=\left\{x \in \mathbb{R}^{n}\right.$ : $A x \leq b\}$ is nonempty, then $C$ is the cone $\left\{x \in \mathbb{R}^{n}: A x \leq 0\right\}$, which is called the recession cone of $P$ and denoted by $\operatorname{rec}(P)$. We will prove this theorem in Section 2.3.

Using the Minkowski-Weyl theorem, we will prove the fundamental theorem of integer programming, due to Meyer [38]:

Given rational matrices $A, G$ and a rational vector $b$, let $P:=\{(x, y)$ : $A x+G y \leq b\}$ and $S:=\{(x, y) \in P: x$ integral $\}$. Then there exist rational matrices $A^{\prime}, G^{\prime}$ and a rational vector $b^{\prime}$ such that $\operatorname{conv}(S)=\left\{(x, y): A^{\prime} x+\right.$ $\left.G^{\prime} y \leq b^{\prime}\right\}$.

In other words, when $P$ is a rational polyhedron, then the convex hull of $S$ is also a rational polyhedron. Note that, if matrices $A, G$ are not rational, then $\operatorname{conv}(S)$ may not be a polyhedron as shown by the following example $S:=\left\{x \in \mathbb{Z}^{2}: 1 \leq x_{2} \leq \sqrt{2} x_{1}\right\}$. In this case, infinitely many inequalities are required to describe $\operatorname{conv}(S)$ by a system of linear inequalities. In this survey we assume that the data $A, G, b$ are rational. Meyer's theorem is the theoretical underpinning of the polyhedral approach to integer programming. Indeed, it shows that (1), the problem of optimizing a linear function over a mixed integer set $S$ is equivalent to solving a linear program. The 
main difficulty is that the polyhedron $\operatorname{conv}(S)$ is not known explicitly. In the later sections of the tutorial, we will address the constructive aspects of $\operatorname{conv}(S)$.

\subsection{Farkas' lemma and linear programming duality}

The following is a fundamental fact in linear algebra:

Theorem 2.1. A system of linear equations $A x=b$ is infeasible if and only if the system $u A=0, u b<0$ is feasible.

A constructive proof of Theorem 2.1 is straightforward using Gaussian elimination on the system $A x=b$. Furthermore, one can decide in polynomial time which of the two systems is feasible, and find a solution, again using Gaussian elimination (Edmonds [24], see e.g. Schrijver [45] p.27). Farkas' lemma [26] provides a certificate of the solvability of a system of linear inequalities $A x \leq b$ in the same spirit as Theorem 2.1. However its proof is not as straightforward. This is not surprising since checking feasibility of a system of linear inequalities is a linear programming problem. In fact, Farkas' lemma can be used to derive the strong duality theorem of linear programming, as we will show later in this section. We first give a proof of Farkas' lemma based on Theorem 2.1, following Conforti, Di Summa and Zambelli [15]. A linear system $a_{i} x=b_{i}, i=1, \ldots, p, \quad a_{i} x \leq b_{i}, i=p+1, \ldots, m$ is minimally infeasible if it has no solution but each of the $m$ linear systems obtained by removing a single constraint has a solution.

Theorem 2.2. (Farkas' lemma) The system of linear inequalities $A x \leq b$ is infeasible if and only if the system $u A=0, u b<0, u \geq 0$ is feasible.

Proof. Assume $u A=0, u b<0, u \geq 0$ is feasible. Then $0=u A x \leq u b<0$ for any $x$ satisfying $A x \leq b$. It follows that $A x \leq b$ is infeasible and this proves the "if" part.

Now we prove the "only if" part. Let $A^{\prime} x \leq b^{\prime}$ be an infeasible system. Let $a_{i}, i \in R$, denote the rows of $A^{\prime}$. Remove inequalities from the system $A^{\prime} x \leq b^{\prime}$ until it becomes minimally infeasible. Let $A x \leq b$ be the resulting system and let $M \subseteq R$ index the rows of $A$. We will show that there exists $u \geq 0$ such that $u A=0$ and $u b<0$. Setting $u_{i}=0$ for all $i \in R \backslash M$, this will show that there exists $u \geq 0$ such that $u A^{\prime}=0$ and $u b^{\prime}<0$, proving the "only if" part. Given $S \subseteq M$, define $\bar{S}:=M \backslash S$. 
Claim. For every $S \subseteq M$ the system $a_{i} x=b_{i}, i \in S, a_{i} x \leq b_{i}, i \in \bar{S}$ is minimally infeasible.

The proof is by induction on $|S|$. The claim is true when $S=\emptyset$. Consider $S \subseteq M$ with $|S| \geq 1$ and assume by induction that the claim holds for any set of smaller cardinality than $|S|$.

The system $a_{i} x=b_{i}, i \in S, a_{i} x \leq b_{i}, i \in \bar{S}$ is infeasible. Let $k \in M$. By the induction hypothesis applied to $S \backslash\{k\}$, the system

$$
a_{i} x=b_{i}, i \in S \backslash\{k\}, a_{i} x \leq b_{i}, i \in \bar{S}
$$

is feasible for any $k \in S$. Therefore, to prove the claim, we only need to show that

$$
a_{i} x=b_{i}, i \in S, \quad a_{i} x \leq b_{i}, i \in \bar{S} \backslash\{k\}
$$

is feasible for any $k \in \bar{S}$. Let $h \in S$. By induction, there exist $x^{h}$ and $x^{k}$ such that

$$
a_{i} x^{h}=b_{i}, i \in S \backslash\{h\}, a_{i} x^{h} \leq b_{i}, i \in \bar{S}
$$

and

$$
a_{i} x^{k}=b_{i}, i \in S \backslash\{h\}, \quad a_{i} x^{k} \leq b_{i}, i \in \bar{S} \cup\{h\} \backslash\{k\} .
$$

Notice that $a_{h} x^{k} \leq b_{h}$ and $a_{h} x^{h}>b_{h}$, so, setting $\alpha=a_{h} x^{h}-b_{h}>0$ and $\beta=b_{h}-a_{h} x^{k} \geq 0$, the vector $y=\frac{\alpha}{\alpha+\beta} x^{k}+\frac{\beta}{\alpha+\beta} x^{h}$ is a solution for (5). This proves the claim.

Now, since $a_{i} x \leq b_{i}, i \in M$, is infeasible, then clearly $a_{i} x=b_{i}, i \in M$, is infeasible and by Theorem 2.1, there exists a vector $u$ such that $u A=$ $0, u b<0$. The lemma holds if $u \geq 0$. So suppose $u_{1}<0$. According to the Claim, there is a vector $x^{*}$ such that $a_{i} x^{*}=b_{i}, i \in M \backslash\{1\}$. Since $A x \leq b$ is an infeasible system, $a_{1} x^{*}>b_{1}$. This (together with $u_{1}<0$ ) shows $u\left(A x^{*}-b\right)<0$, contradicting

$$
u\left(A x^{*}-b\right)=(u A) x^{*}-u b>0
$$

where the inequality follows from $u A=0$ and $u b<0$.

Equivalently, Farkas' lemma can be written as:

Corollary 2.3. The system of linear inequalities $A x \leq b$ is feasible if and only if $u b \geq 0$ for every vector $u$ satisfying $u A=0, u \geq 0$.

Farkas' lemma can also be restated as follows:

Theorem 2.4. The system $A x=b, x \geq 0$ is feasible if and only if $u b \geq 0$ for every $u$ satisfying $u A \geq 0$. 
Proof. The system $A x=b, x \geq 0$ is equivalent to $A x \leq b,-A x \leq$ $-b,-x \leq 0$. The theorem follows by applying Corollary 2.3 to this latter system.

The following is a more general, yet still equivalent, form of Farkas' lemma.

Theorem 2.5. The system $A x+B y \leq f, C x+D y=g, x \geq 0$ is feasible if and only if $u f+v g \geq 0$ for every $(u, v)$ satisfying $u A+v C \geq 0, u B+v D=$ $0, u \geq 0$.

Checking the feasibility of a system of linear inequalities can be done in polynomial time (Khachiyan [34], Karmarkar [33]). Next we derive the fundamental theorem of Linear Programming from Farkas' lemma.

Theorem 2.6. (Linear Programming Duality) Let

$$
P:=\{x: A x \leq b\} \quad \text { and } D:=\{u: u A=c, u \geq 0\} .
$$

If $P$ and $D$ are both nonempty, then

$$
\max \{c x: x \in P\}=\min \{u b: u \in D\} .
$$

Proof. For $\bar{x} \in P$ and $\bar{u} \in D$ we have that $c \bar{x}=\bar{u} A \bar{x} \leq \bar{u} b$. Therefore $" \max \leq \min "$ always holds. To prove equality, we need to prove that the system

$$
-c x+b^{T} u^{T} \leq 0, A x \leq b, A^{T} u^{T}=c^{T}, u^{T} \geq 0
$$

is feasible. By Farkas' lemma (Theorem 2.5), (7) is feasible if and only $\mu b+\nu c^{T} \geq 0$ for every vector $(\lambda, \mu, \nu)$ satisfying

$$
\mu A-\lambda c=0, \quad \nu A^{T}+\lambda b^{T} \geq 0, \quad \lambda, \mu \geq 0 .
$$

If $\lambda>0$, then $\mu b=\lambda^{-1} \lambda b^{T} \mu^{T} \geq-\lambda^{-1} \nu A^{T} \mu^{T}=-\lambda^{-1} \nu c^{T} \lambda=-\nu c^{T}$.

If $\lambda=0$, let $\bar{x} \in P$ and $\bar{u} \in D$. Then $\mu b \geq \mu A \bar{x}=0 \geq-\nu A^{T} u^{T}=-\nu c^{T}$. Therefore in both cases we have $\mu b+\nu c^{T} \geq 0$ and the proof is complete.

Theorem 2.7. (Complementary slackness) Let $X^{\text {opt }}$ and $U^{\text {opt }}$ be subsets of $P:=\{x: A x \leq b\}$ and $D:=\{u: u A=c, u \geq 0\}$ respectively. Define $I=\left\{i: u_{i}>0\right.$ for some $\left.u \in U^{\text {opt }}\right\}$.

Then $X^{\text {opt }}$ and $U^{\text {opt }}$ are the sets of optimal solutions of the pair of dual LPs

$$
\max \{c x: x \in P\} \quad \text { and } \min \{u b: u \in D\}
$$

if and only if

$$
X^{o p t}=\left\{x: a_{i} x=b_{i}, i \in I, a_{i} x \leq b_{i}, i \notin I\right\} .
$$


Proof. By Theorem 2.6, the sets $X^{\text {opt }}$ and $U^{\text {opt }}$ are the sets of optimal solutions of the pair of dual LPs above if and only if $c x=u b$ for every $x \in X^{o p t}$, $u \in U^{o p t}$. For every $x \in P$ and $u \in D, c x=u A x \leq u b$, hence equality holds throughout if and only if $a_{i} x=b_{i}$ for every $i \in I$ and every $x \in X^{o p t}$.

Here is another well-known consequence of Farkas' lemma:

Remark 2.8. Let $P:=\{x: A x \leq b\}$ and $D:=\{u: u A=c, u \geq 0\}$. If $D=\emptyset$ and $P \neq \emptyset$, then $\max \{c x: x \in P\}$ is unbounded.

Proof. Since $D=\emptyset$, by Farkas' lemma, the system $A y \leq 0, c y>0$ is feasible: Let $\bar{y}$ be a solution of this system and $\bar{x} \in P$. Then $\bar{x}+\lambda \bar{y} \in P$ for every $\lambda \geq 0$. Since $c \bar{y}>0, \max \{c x: x \in P\}$ is unbounded.

\subsection{Caratheodory's theorem}

Theorem 2.9. (Caratheodory's theorem) If the linear system $A x=b, x \geq 0$ is feasible, then it has a solution $\bar{x}$ where the columns of $A$ corresponding to the positive entries of $\bar{x}$ are linearly independent.

Proof. Let $\bar{x}$ be a solution with the minimum number of positive entries and let $\bar{A}$ be the column submatrix of $A$ corresponding to the positive entries of $\bar{x}$. If the columns of $\bar{A}$ are linearly dependent, there exists a vector $y \neq 0$ such that $A y=0$ and $y_{j}=0$ whenever $\bar{x}_{j}=0$. We assume w.l.o.g. that $y$ has at least one positive component. Let $\lambda=\min _{y_{j}>0} \frac{\bar{x}_{j}}{y_{j}}$, let $j^{*}$ be an index for which this minimum is attained and define $x^{*}=\bar{x}-\lambda y$. Clearly $A x^{*}=b$. By the choice of $\lambda, x^{*} \geq 0$ and $x_{j}^{*}=0$ whenever $\bar{x}_{j}=0$. Furthermore $x_{j^{*}}^{*}=0$ while $\bar{x}_{j^{*}}>0$, a contradiction to our choice of $\bar{x}$.

The next theorem combines Caratheodory's Theorem 2.9 with a strengthening of Farkas' lemma.

Theorem 2.10. For a linear system $A x=b, x \geq 0$ exactly one of the following two alternatives holds:

- $A x=b, x \geq 0$ admits a solution $\bar{x}$ where the columns of $A$, corresponding to the positive entries of $\bar{x}$, are linearly independent.

- There is a vector $u$ such that $u A \geq 0, u b<0$ and there is a column submatrix $A^{0}$ of $A$ such that $u A^{0}=0$ and $\operatorname{rank}\left(A^{0}\right)=\operatorname{rank}(A \mid b)-1$. 
Proof. If $A x=b, x \geq 0$ admits a solution, then Theorem 2.9 shows that the first outcome holds. So we assume that $A x=b, x \geq 0$ is infeasible and we show that the second outcome holds.

If $\operatorname{rank}(A)=\operatorname{rank}(A \mid b)-1$, then by standard linear algebra $A x=b$ is infeasible, so by Theorem 2.1 there exists a vector $u$ such that $u A=0$, $u b<0$ and the theorem obviously holds in this case.

So we consider the case $\operatorname{rank}(A)=\operatorname{rank}(A \mid b)$ and we can assume that $A$ has full row-rank $m$. By Theorem 2.4, there exists a vector $u$ such that $u A \geq 0$, $u b<0$. Among such vectors, choose $u$ such that the column submatrix $A^{0}$ of $A$ where $u A^{0}=0$ has maximum rank. Suppose by contradiction that $\operatorname{rank}\left(A^{0}\right) \leq m-2$. Then $\operatorname{rank}\left(A^{0} \mid A \mathbf{1}\right) \leq m-1$ where $\mathbf{1}$ denotes the vector of all 1s. So there exists a vector $v \neq 0$ such that $v A^{0}=0, v A \mathbf{1}=0$, and we may choose $v$ such that $v b \geq 0$.

Let $J$ be the set of column-indices of $A=\left(a^{1}, \ldots, a^{n}\right)$ and $J^{0}$ be the subset of $J$, corresponding to the column indices of $A^{0}$. Since $v \neq 0$ and $A$ has full row-rank, there is an index $j$ such that $v a^{j} \neq 0$. Since $v A \mathbf{1}=0$, then there is an index $j$ such that $v a^{j}>0$. (Note that such an index $j$ is in $J \backslash J^{0}$ ).

Let $\lambda=\min _{j \in J: v a^{j}>0} \frac{u a^{j}}{v a^{j}}$ and let $j^{*}$ be an index for which this minimum is attained. Then by the choice of $\lambda,(u-\lambda v) A \geq 0$ and $(u-\lambda v) b<$ 0 . Furthermore $(u-\lambda v) A^{0}=0$ and $(u-\lambda v) a^{j^{*}}=0$. Since $v A^{0}=0$ and $v a^{j^{*}}>0$, then $A^{0} x=a^{j^{*}}$ is infeasible by Theorem 2.1. This implies $\operatorname{rank}\left(A^{0} \mid a^{j^{*}}\right)=\operatorname{rank}\left(A^{0}\right)+1$. Therefore $u-\lambda v$ contradicts the choice of $u$.

\subsection{The theorem of Minkowski-Weyl}

We first present the Minkowski-Weyl theorem for cones.

Theorem 2.11. (Minkowski-Weyl theorem for cones) For a set $C \subseteq \mathbb{R}^{n}$, the two following conditions are equivalent:

1. There is a matrix $A$ such that $C=\left\{x \in \mathbb{R}^{n}: A x \geq 0\right\}$.

2. There is a matrix $R$ such that $C=\left\{x \in \mathbb{R}^{n}: x=R \mu, \mu \geq 0\right\}$.

In the terminology introduced in Section 2, Theorem 2.11 states that a cone is polyhedral if and only if it is finitely generated. The columns of $R$ are the generators of the cone $C$.

We first show that any one of the two conditions of the theorem is implied by the other. A pair of matrices $(A, R)$ that satisfy $\left\{x \in \mathbb{R}^{n}: A x \geq 0\right\}=$ $\left\{x \in \mathbb{R}^{n}: x=R \mu, \mu \geq 0\right\}$ will be called an $M W$-pair. Note that if $(A, R)$ 
is an MW-pair, then all entries of $A R$ are nonnegative (since $A R e^{j} \geq 0$ for the unit vectors $e^{j}$ ).

Lemma 2.12. A pair of matrices $(A, R)$ is an $M W$-pair if and only if $\left(R^{T}, A^{T}\right)$ is an $M W$-pair.

Proof. By Farkas' lemma (Theorem 2.4), $\left\{x \in \mathbb{R}^{n}: x=R \mu, \mu \geq 0\right\}=$ $\left\{x \in \mathbb{R}^{n}: x^{T} y \geq 0\right.$ for every $y$ satisfying $\left.R^{T} y \geq 0\right\}$. Therefore $(A, R)$ is an MW-pair if and only if:

$$
\{x: A x \geq 0\}=\left\{x \in \mathbb{R}^{n}: x^{T} y \geq 0 \text { for every } y \text { s.t. } R^{T} y \geq 0\right\} .
$$

Assuming that (8) holds, we show that $\left\{y: R^{T} y \geq 0\right\}=\left\{y: y=A^{T} \mu, \mu \geq\right.$ $0\}$. This is the condition for $\left(R^{T}, A^{T}\right)$ to be an MW-pair.

Given $\bar{y}$ such that $R^{T} \bar{y} \geq 0$, then by (8), $\bar{y}^{T} x \geq 0$ for every $x$ satisfying $A x \geq 0$. By Farkas' Lemma (Theorem 2.4) the system $\bar{y}=A^{T} \mu, \mu \geq 0$ is feasible. This shows that $\left\{y: R^{T} y \geq 0\right\} \subseteq\left\{y: y=A^{T} \mu, \mu \geq 0\right\}$.

We show the reverse inclusion. Given $\bar{y}$ such that $\bar{y}=A^{T} \mu$ for some $\mu \geq 0$, $R^{T} \bar{y}=R^{T} A^{T} \mu \geq 0$, because all entries of $A R$ are nonnegative since $(A, R)$ is an MW-pair.

Proof of Theorem 2.11

By Lemma 2.12 it is enough to prove that 2 implies 1.

Let $r^{1}, \ldots, r^{k}$ be the columns of $R$. We may assume that the vectors $r^{1}, \ldots, r^{k}$ span $\mathbb{R}^{n}$, else all these vectors satisfy an equation $d r=0$ where $d \neq 0$ and one variable can be eliminated (i.e. the dimension can be reduced). Now consider the half spaces $\left\{x \in \mathbb{R}^{n}: a x \geq 0\right\}$ that contain $\left\{r^{1}, \ldots, r^{k}\right\}$ such that the hyperplane $\left\{x \in \mathbb{R}^{n}: a x=0\right\}$ contains $n-1$ linearly independent vectors among $r^{1}, \ldots, r^{k}$. Since these vectors span $\mathbb{R}^{n}$, there is a finite number of such half-spaces. (In fact, at most $\left(\begin{array}{c}k \\ n-1\end{array}\right)$ ). Let $A$ be the matrix whose rows contain the incidence vector $a$ of all such subspaces and consider the cones

$$
C_{A}:=\left\{x \in \mathbb{R}^{n}: A x \geq 0\right\}, \quad C_{R}:=\left\{x \in \mathbb{R}^{n}: x=R \mu, \mu \geq 0\right\} .
$$

Since every inequality of the system $A x \geq 0$ is valid for $r^{1}, \ldots, r^{k}, C_{R} \subseteq$ $C_{A}$.

Let $\bar{x} \notin C_{R}$. Then the system $\bar{x}=R \mu, \mu \geq 0$ is infeasible. By Theorem 2.10, there exists $u$ such that $u R \geq 0, u \bar{x}<0$ and there exists a column submatrix $R^{0}$ of $R$ such that $u R^{0}=0$ and $\operatorname{rank}\left(R^{0}\right)=\operatorname{rank}(R \mid \bar{x})-1=n-1$ (because $r^{1}, \ldots, r^{k}$ span $\mathbb{R}^{n}$ ). Therefore $u$ is one of the vectors $a$ from $A x \geq 0$. But then $a \bar{x}<0$, i.e. $\bar{x} \notin C_{A}$. So $C_{R}$ coincides with $C_{A}$ and the theorem follows. 
Remark 2.13. The proof of Theorem 2.11 shows that, if $A$ is a rational matrix, there exists a rational matrix $R$ such that $(A, R)$ is an $M W$-pair.

We now present the Minkowski-Weyl theorem for polyhedra. Given subsets $V, R$ of $\mathbb{R}^{n}$, the Minkowski sum of $V, R$ is the set:

$$
V+R:=\left\{x \in \mathbb{R}^{n}: \text { there exist } v \in V, r \in R \text { such that } x=v+r\right\} .
$$

If one of $V, R$ is empty, the Minkowski sum of $V, R$ is empty.

Theorem 2.14. (Minkowski-Weyl theorem for polyhedra) For a subset $P$ of $\mathbb{R}^{n}$, the following two conditions are equivalent:

1. $P$ is a polyhedron, i.e. there is a matrix $A$ and a vector $b$ such that $P=\left\{x \in \mathbb{R}^{n}: A x \leq b\right\}$.

2. There are finite sets of vectors $v^{1}, \ldots, v^{p}, r^{1}, \ldots, r^{q}$ such that

$$
P=\operatorname{conv}\left(v^{1}, \ldots, v^{p}\right)+\operatorname{cone}\left(r^{1}, \ldots, r^{q}\right) .
$$

Proof. We show that 1 implies 2. Consider the polyhedral cone $C_{P}:=$ $\left\{(x, y) \in \mathbb{R}^{n+1}: b y-A x \geq 0, y \geq 0\right\}$. Notice that $P=\left\{x:(x, 1) \in C_{P}\right\}$. By Theorem 2.11, the cone $C_{P}$ is finitely generated. Since $y \geq 0$ for every vector $(x, y) \in C_{P}$, we can assume that $y=0$ or 1 for all the rays that generate $C_{P}$. That is,

$$
C_{P}=\text { cone }\left\{\left(\begin{array}{c}
v^{1} \\
1
\end{array}\right), \ldots,\left(\begin{array}{c}
v^{p} \\
1
\end{array}\right),\left(\begin{array}{c}
r^{1} \\
0
\end{array}\right), \ldots,\left(\begin{array}{c}
r^{q} \\
0
\end{array}\right)\right\} .
$$

Therefore $P=\operatorname{conv}\left\{v^{1}, \ldots, v^{p}\right\}+\operatorname{cone}\left\{r^{1}, \ldots, r^{q}\right\}$.

The converse statement follows analogously from Theorem 2.11.

Corollary 2.15. (Minkowski-Weyl theorem for polytopes) For a set $P \subseteq$ $\mathbb{R}^{n}$, the following two conditions are equivalent:

1. $P$ is bounded and there is a matrix $A$ and $a$ vector $b$ such that $P=$ $\left\{x \in \mathbb{R}^{n}: A x \leq b\right\}$.

2. There is a finite set of vectors $v^{1}, \ldots, v^{p}$ such that $P=\operatorname{conv}\left(v^{1}, \ldots, v^{p}\right)$.

For a matrix $V:=\left(v^{1}, \ldots, v^{p}\right)$ whose columns are the vectors $v^{j}$, it will be convenient to denote by $\operatorname{conv}(V)$ the set $\operatorname{conv}\left(v^{1}, \ldots, v^{p}\right)$. Similarly cone $(V)$ will denote the set cone $\left(v^{1}, \ldots, v^{p}\right)$. 


\subsection{Projections}

Let $P \subseteq \mathbb{R}^{n+p}$ where $(x, y) \in P$ will be interpreted as meaning $x \in \mathbb{R}^{n}$ and $y \in \mathbb{R}^{p}$. The projection of $P$ onto the $x$-space $\mathbb{R}^{n}$ is

$$
\operatorname{proj}_{x}(P):=\left\{x \in \mathbb{R}^{n}: \exists y \in \mathbb{R}^{p} \text { with }(x, y) \in P\right\} .
$$

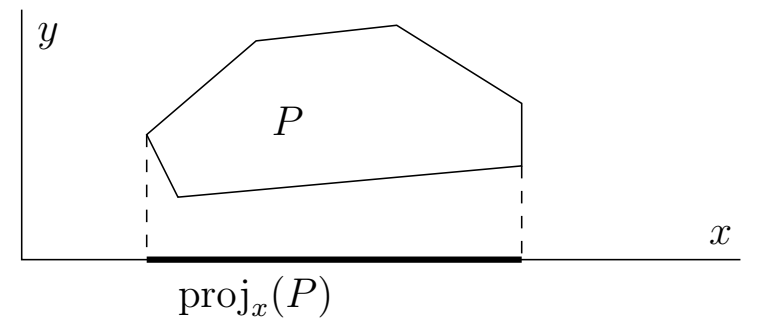

Figure 3: Projection

Theorem 2.16. Let $P:=\left\{(x, y) \in \mathbb{R}^{n} \times \mathbb{R}^{p}: A x+G y \leq b\right\}$. Then $\operatorname{proj}_{x}(P)=\left\{x \in \mathbb{R}^{n}: v^{t}(b-A x) \geq 0\right.$ for all $\left.t \in T\right\}$ where $\left\{v^{t}\right\}_{t \in T}$ is the set of extreme rays of $Q:=\left\{v \in \mathbb{R}^{m}: v G=0, v \geq 0\right\}$.

Proof. Let $x \in \mathbb{R}^{n}$. By Farkas's Lemma, $G y \leq b-A x$ has a solution $y$ if and only if $v^{t}(b-A x) \geq 0$ for all $v \in Q$. Since $v \geq 0$ for every $v \in Q$, then $Q$ is pointed, hence it is generated by its extreme rays, and the statement follows.

Remark 2.17. Variants of Theorem 2.16 can be proved similarly:

If $y \geq 0$ in $P$, then the relevant cone $Q$ is $\{v: v G \geq 0, v \geq 0\}$.

If $y \geq 0$ and $A x+G y=b$ in $P$, the relevant cone is $\{v: v G \geq 0\}$ with $v$ unrestricted in sign.

Enumerating the extreme rays of $Q$ may not be an easy task in applications. Another way of obtaining the projection of $P$ is to eliminate the variables $y_{i}$ one at a time (Fourier elimination procedure):

Consider a polyhedron $P \subseteq \mathbb{R}^{n+1}$ defined by the system of inequalities:

$$
\sum_{j=1}^{n} a_{i j} x_{j}+g_{i} z \leq b_{i} \quad \text { for } i \in I .
$$

Let $I^{0}=\left\{i \in I: g_{i}=0\right\}, I^{+}=\left\{i \in I: g_{i}>0\right\}, I^{-}=\left\{i \in I: g_{i}<0\right\}$. The Fourier procedure eliminates the variable $z$ as follows: It keeps the inequalities of (9) in $I^{0}$, and it combines each pair of inequalities $i \in I^{+}$and $l \in I^{-}$to eliminate $z$. 
Theorem 2.18. The system of $\left|I^{0}\right|+\left|I^{+}\right|\left|I^{-}\right|$inequalities obtained by the Fourier procedure is the projection $\operatorname{proj}_{x}(P)$ of $P$ in the $x$-space $\mathbb{R}^{n}$.

We leave the proof as a (fairly easy) exercise to the reader.

\subsection{The fundamental theorem for MILP}

Let $S:=\{(x, y): A x+G y \leq b, x$ integral $\}$ be a mixed integer set, where matrices $A, G$ and vector $b$ have rational entries. Meyer [38] proved that $\operatorname{conv}(S)$ is a rational polyhedron, i.e. there exist rational matrices $A^{\prime}$ and $G^{\prime}$ and a rational vector $b^{\prime}$ such that $\operatorname{conv}(S)=\left\{(x, y): A^{\prime} x+G^{\prime} y \leq b^{\prime}\right\}$.

Note first that if $S$ contains finitely many vectors (for instance this happens when $S$ is the set of integral vectors contained in a polytope), the above result follows from Corollary 2.15, without the need for the assumption that $A, G$ and $b$ have rational entries.

Theorem 2.19. (Meyer [38]) Given rational matrices $A, G$ and a rational vector $b$, let $P:=\{(x, y): A x+G y \leq b\}$ and let $S:=\{(x, y) \in P$ : $x$ integral\}.

1. There exist rational matrices $A^{\prime}, G^{\prime}$ and a rational vector $b^{\prime}$ such that $\operatorname{conv}(S)=\left\{(x, y): A^{\prime} x+G^{\prime} y \leq b^{\prime}\right\}$.

2. If $S$ is nonempty, the recession cones of $\operatorname{conv}(S)$ and $P$ coincide.

Proof. If $S=\emptyset$, the existence of rational $A^{\prime}, G^{\prime}$ and $b^{\prime}$ satisfying the theorem is obvious. So we assume that $S$ and $P$ are both nonempty. By the Minkowski-Weyl theorem (Theorem 2.14), $P=\operatorname{conv}(V)+\operatorname{cone}(R)$ where $V=\left(v^{1}, \ldots, v^{p}\right), R=\left(r^{1}, \ldots, r^{q}\right)$. Since $A, G$ and $b$ are rational, by Remark 2.13 we can assume that $V$ is a rational matrix and $R$ is an integral matrix.

Consider the following truncation of $P$

$$
T:=\left\{(x, y):(x, y)=\sum_{i=1}^{p} \lambda_{i} v^{i}+\sum_{j=1}^{q} \mu_{j} r^{j}, \sum_{i=1}^{p} \lambda_{i}=1, \lambda \geq 0,0 \leq \mu \leq \mathbf{1}\right\} .
$$

$T$ is bounded and is the projection of a rational polyhedron. Therefore it is a rational polytope (Corollary 2.15). Let $T_{I}:=\{(x, y) \in T: x$ integral $\}$.

Claim. $\operatorname{conv}\left(T_{I}\right)$ is a rational polytope.

Since $T$ is a polytope, the set $X:=\left\{x:\right.$ there exist $y$ s.t. $\left.(x, y) \in T_{I}\right\}$ is a finite set. For fixed $\bar{x} \in X$, the set $T_{\bar{x}}:=\left\{(\bar{x}, y):(\bar{x}, y) \in T_{I}\right\}$ is a 
rational polytope and therefore by Corollary $2.15, T_{\bar{x}}=\operatorname{conv}\left(V_{\bar{x}}\right)$ for some rational matrix $V_{\bar{x}}$. Since $X$ is a finite set, there is a rational matrix $V_{T_{I}}$ which contains all the columns of all the matrices $V_{\bar{x}}$, for $\bar{x} \in X$. Therefore $\operatorname{conv}\left(T_{I}\right)=\operatorname{conv}\left(V_{T_{I}}\right)$ and this proves the claim.

A point $(\bar{x}, \bar{y})$ belongs to $S$ if and only if $\bar{x}$ is integral and there exist multipliers $\lambda \geq 0, \sum_{i=1}^{p} \lambda_{i}=1$ and $\mu \geq 0$ such that

$$
(\bar{x}, \bar{y})=\sum_{i=1}^{p} \lambda_{i} v^{i}+\sum_{j=1}^{q}\left(\mu_{j}-\left\lfloor\mu_{j}\right\rfloor\right) r^{j}+\sum_{j=1}^{q}\left\lfloor\mu_{j}\right\rfloor r^{j} .
$$

The point $\sum_{i=1}^{p} \lambda_{i} v^{i}+\sum_{j=1}^{q}\left(\mu_{j}-\left\lfloor\mu_{j}\right\rfloor\right) r^{j}$ belongs to $T$ and therefore it also belongs to $T_{I}$ since $\bar{x}$ and $\sum_{j=1}^{q}\left\lfloor\mu_{j}\right\rfloor r^{j}$ are integral vectors. Thus

$$
S=T_{I}+R_{I}
$$

where $R_{I}$ is the set of integral conic combinations of $r^{1}, \ldots, r^{q}$.

This shows in particular that $T_{I}$ is nonempty. Equation (10) implies that $\operatorname{conv}(S)=\operatorname{conv}\left(T_{I}\right)+\operatorname{cone}(R)$. By the above claim, $\operatorname{conv}\left(T_{I}\right)$ is a rational polytope. Thus $\operatorname{conv}(S)=\operatorname{conv}\left(T_{I}\right)+\operatorname{cone}(R)$ is a rational polyhedron having the same recession cone (namely cone $(R)$ ) as $P$.

Remark 2.20. In Theorem 2.19:

- If matrices $A, G$ are not rational, then $\operatorname{conv}(S)$ may not be a polyhedron. One such example is the set $S:=\left\{x \in \mathbb{Z}^{2}: 1 \leq x_{2} \leq \sqrt{2} x_{1}\right\}$.

- If $A, G$ are rational matrices but $b$ is not rational, then $\operatorname{conv}(S)$ is a polyhedron that has the same recession cone as $P$. However $\operatorname{conv}(S)$ is not always a rational polyhedron. (This can be inferred from the above proof).

\subsection{Valid Inequalities}

An inequality $c x \leq \delta$ is valid for the set $P \subseteq \mathbb{R}^{n}$ if $c x \leq \delta$ is satisfied by every point in $P$.

Theorem 2.21. Let $P:=\{x: A x \leq b\}$ be a nonempty polyhedron. An inequality $c x \leq \delta$ is valid for $P$ if and only if the system $u A=c, u b \leq \delta, u \geq 0$ is feasible. 
Proof. Consider the linear program $\max \{c x: x \in P\}$. Since $P \neq \emptyset$ and $c x \leq \delta$ is a valid inequality for $P$, the above program admits a finite optimum and its value is $\delta^{\prime} \leq \delta$.

By Remark 2.8, the set $D=\{u: u A=c, u \geq 0\}$ is nonempty. Therefore by Theorem 2.6, $\delta^{\prime}$ is the common value of the equation (6). Thus an optimum solution $u$ of $\min \{u b: u \in D\}$ satisfies $u A=c, u b \leq \delta, u \geq 0$.

Conversely, assume $u A=c, u b \leq \delta, u \geq 0$ is feasible. Then, for all $x \in P$, we have $c x=u A x \leq u b \leq \delta$. This shows that $c x \leq \delta$ is valid for $P$.

\section{$2.7 \quad$ Facets}

Let $P:=\left\{x \in \mathbb{R}^{n}: A x \leq b\right\}$ be a polyhedron. A face of $P$ is a set of the form

$$
F:=P \cap\left\{x \in \mathbb{R}^{n}: c x=\delta\right\}
$$

where $c x \leq \delta$ is a valid inequality for $P$ (the inequality is said to define the face $F$ ). A face is itself a polyhedron since it is the intersection of the polyhedron $P$ with another polyhedron (the hyperplane $c x=\delta$ ). A face of $P$ is said proper if it is nonempty and properly contained in $P$. Maximal proper faces of $P$ are called facets.

Theorem 2.22. Let $P:=\left\{x \in \mathbb{R}^{n}: A x \leq b\right\}$ be a nonempty polyhedron. Let $M$ be the set of row indices of $A$ and let $I \subseteq M$. The set

$$
F_{I}:=\left\{x \in \mathbb{R}^{n}: a_{i} x=b_{i}, i \in I, a_{i} x \leq b_{i}, i \in M \backslash I\right\} .
$$

is a face of $P$. Conversely, if $F$ is a nonempty face of $P$, then $F=F_{I}$ for some $I \subseteq M$.

Proof. Let $u$ be a vector such that $u_{i}>0, i \in I, u_{i}=0, i \in M \backslash I$, and let $c:=u A, \delta:=u b$. Then, given $x \in P$, clearly $x$ satisfies $c x=\delta$ if and only if it satisfies $a_{i} x=b_{i}, i \in I$. Thus $F_{I}=P \cap\left\{x \in \mathbb{R}^{n}: c x=\delta\right\}$, so $F_{I}$ is a face.

Conversely, let $F:=\{x \in P: c x=\delta\}$ be a nonempty face of $P$. Then $F$ is the set of optimal solutions of the LP $\max \{c x: x \in P\}$. Let $I$ be the set of indices defined as in Theorem 2.7. Then by Theorem 2.7, $F=F_{I}$.

Theorem 2.22 has important consequences:

- The number of faces of $P$ is finite. 
- The intersection of faces of $P$ is a face of $P$.

- If $F$ is a face of $P$ and $F^{\prime}$ is a face of $F$, then $F^{\prime}$ is also a face of $P$.

- If $F_{1}, F_{2}$ are faces of $P$, there is a unique minimal face $F$ of $P$ that contains both $F_{1}$ and $F_{2}$ (The system of equalities that defines $F$ is the intersection of the systems of equalities that define $F_{1}$ and $F_{2}$ ).

Furthermore, by Theorem 2.22, we can express a face $F$ of $P$ as follows. Let $A_{F}^{\bar{F}} x \leq b_{\bar{F}}$ be the set of all the inequalities $a_{i} x \leq b_{i}$ in $A x \leq b$ such that $F \subseteq\left\{x \in \mathbb{R}^{n}: a_{i} x=b_{i}\right\}$. Then

$$
F=P \cap\left\{x \in \mathbb{R}^{n}: A_{\bar{F}}^{\overline{\bar{F}}} x=b_{\bar{F}}^{\overline{\bar{F}}}\right\} .
$$

An inequality $a_{i} x \leq b_{i}$ from $A x \leq b$ such that $a_{i} x=b_{i}$ for all $x \in P$ is called an implicit equality of $P$. Let us partition the inequalities $A x \leq b$ defining $P$ into the implicit equalities $A^{=} x \leq b^{=}$and the rest $A^{<} x \leq b^{<}$ (either of these two families of inequalities could be empty). Thus $P=\{x \in$ $\left.\mathbb{R}^{n}: A^{=} x=b^{=}, A^{<} x \leq b^{<}\right\}$and for each inequality $a_{i} x \leq b_{i}$ in $A^{<} x \leq b^{<}$, there exists $\bar{x} \in P$ such that $a_{i} \bar{x}<b_{i}$.

Remark 2.23. $P$ contains a point $\bar{x}$ such that $A^{=} \bar{x}=b^{=}, A^{<} \bar{x}<b^{<}$.

Proof. Indeed, for every inequality $a_{i} x \leq b_{i}$ in $A^{<} x \leq b^{<}$, there is a point $x^{i} \in P$ such that $a_{i} x^{i}<b_{i}$. Let $r$ be the number of these points. Then $\bar{x}:=\frac{1}{r} \sum_{i=1}^{r} x^{i}$ satisfies the statement.

An inequality $a_{i} x \leq b_{i}$ of the system $A x \leq b$ is redundant if $a_{i} x \leq b_{i}$ is a valid inequality for the system obtained from $A x \leq b$ by removing the inequality $a_{i} x \leq b_{i}$. Theorem 2.21 provides a characterization of redundant inequalities.

Let $I^{<}$denote the set of indices of the rows of $A^{<} x \leq b^{<}$. For every $i \in I^{<}$, denote by $A_{-i}^{<} x \leq b_{-i}^{<}$the system obtained from $A^{<} x \leq b^{<}$by removing the inequality $a_{i}^{<} x \leq b_{i}^{<}$.

Lemma 2.24. Assume that $A^{<} x \leq b^{<}$contains no redundant inequality. Then for every $i \in I^{<}$, the polyhedron $P$ contains a point $x^{i}$ satisfying

$$
A^{=} x=b^{=}, \quad A_{-i}^{<} x<b_{-i}^{<}, \quad a_{i}^{<} x=b_{i}^{<} .
$$

Proof. Let $i \in I^{<}$. Since no inequality in $A^{<} x \leq b^{<}$is redundant, the system:

$$
A^{=} x=b^{=}, \quad A_{-i}^{<} x \leq b_{-i}^{<}, \quad a_{i}^{<} x>b_{i}^{<}
$$


is feasible. Let $\bar{x}^{i}$ be a point satisfying this system. By Remark 2.23, there is a point $\bar{x}$ satisfying $A^{=} x=b^{=}, A^{<} x<b^{<}$. Then a point on the segment having $\bar{x}$ and $\bar{x}^{i}$ as endpoints satisfies the above property.

Theorem 2.25. Let $P \subseteq \mathbb{R}^{n}$ be a polyhedron. Partition the inequalities defining $P$ into the implicit equalities and the rest $P=\left\{x \in \mathbb{R}^{n}: A^{=} x=\right.$ $\left.b^{=}, A^{<} x \leq b^{<}\right\}$. Assume that $A^{<} x \leq b^{<}$contains no redundant inequality. Then the facets of $P$ are the elements in the family:

$$
\mathcal{F}=\left\{F_{i}:=\left\{x \in P, a_{i}^{<} x=b_{i}^{<}\right\}, i \in I^{<}\right\} .
$$

Proof. Let $F$ be a facet of $P$. Since no inequality in $A^{<} x \leq b^{<}$is an implicit equality for $P$, by Theorem 2.22 and the maximality of $F$, we have that $F=F_{i}$, for some $i \in I^{<}$. Therefore $\mathcal{F}$ contains all the facets of $P$.

Conversely, let $F_{i} \in \mathcal{F}$. Then by Lemma $2.24, F_{i}$ is a proper face of $P$ and it is not contained in any other face $F_{j} \in \mathcal{F}$. Since $\mathcal{F}$ contains all the facets of $P, F_{i}$ is a proper face of $P$ that is maximal with respect to inclusion, i.e. $F_{i}$ a facet of $P$.

This result states that, if a polyhedron in $\mathbb{R}^{n}$ has $m$ facets, any representation by a system of linear inequalities in $\mathbb{R}^{n}$ contains at least $m$ inequalities. In integer linear programming, we often consider polyhedra that are given implicitly as $\operatorname{conv}(S)$. It is not unusual for such polyhedra to have a number of facets that is exponential in the size of the input. Thus their representation by linear inequalities in $\mathbb{R}^{n}$ is large. In some cases, there is a way to get around this difficulty: a polyhedron with a large number of facets can sometimes be obtained as the projection of a polyhedron with a small number of facets. We illustrate this idea in the next section.

\section{Union of Polyhedra}

In this section, we prove a result of Balas [2, 3] about the union of $k$ polyhedra. Consider $k$ polyhedra $P_{i}:=\left\{x \in \mathbb{R}^{n}: A_{i} x \leq b^{i}\right\}, i=1, \ldots, k$ and their union $\cup_{i=1}^{k} P_{i}$. We will show that $\overline{\operatorname{conv}}\left(\cup_{i=1}^{k} P_{i}\right)$, the smallest closed convex set that contains $\cup_{i=1}^{k} P_{i}$, is a polyhedron. Furthermore we will show that this polyhedron can be obtained as the projection onto $\mathbb{R}^{n}$ of a polyhedron with polynomially many variables and constraints in a higher-dimensional space.

The closure is needed as shown by the following example: $P_{1}$ consists of a single point, and $P_{2}$ is a line that does not contain the point $P_{1}$ (see 
Figure 4). Let $P_{3}$ denote the line going through $P_{1}$ that is parallel to $P_{2}$. It is easy to verify that $\overline{\operatorname{conv}}\left(P_{1} \cup P_{2}\right)=\operatorname{conv}\left(P_{2} \cup P_{3}\right)$ and that $\operatorname{conv}\left(P_{1} \cup P_{2}\right)=$ $\operatorname{conv}\left(P_{2} \cup P_{3}\right) \backslash\left(P_{3} \backslash P_{1}\right)$ (indeed, a point $x^{*}$ in $P_{3} \backslash P_{1}$ is not in $\operatorname{conv}\left(P_{1} \cup P_{2}\right)$, but there is a sequence of points $x^{k} \in \operatorname{conv}\left(P_{1} \cup P_{2}\right)$ that converges to $\left.x^{*}\right)$. Here $\operatorname{conv}\left(P_{1} \cup P_{2}\right)$ is not a closed set, and therefore it is not a polyhedron.



Figure 4: $\overline{\operatorname{conv}}\left(P_{1} \cup P_{2}\right) \neq \operatorname{conv}\left(P_{1} \cup P_{2}\right)$

We recall that, by Minkowski-Weil's Theorem 2.14, $P_{i}=Q_{i}+C_{i}$, where $Q_{i}$ is a polytope and $C_{i}$ a finitely generated cone.

Theorem 3.1. Let $P_{i}=Q_{i}+C_{i}$ be nonempty polyhedra $i=1, \ldots, k$. Then $Q=\operatorname{conv}\left(\cup_{i=1}^{k} Q_{i}\right)$ is a polytope, $C=\operatorname{conv}\left(\cup_{i=1}^{k} C_{i}\right)$ is a finitely generated cone, and $\overline{\mathrm{conv}}\left(\cup_{i=1}^{k} P_{i}\right)$ is the polyhedron

$$
\overline{\operatorname{conv}}\left(\cup_{i=1}^{k} P_{i}\right)=Q+C .
$$

Proof. We first show that $Q$ is a polytope and $C$ a finitely generated cone. For $i=1, \ldots, k$, let $V_{i}$ and $C_{i}$ be finite sets in $\mathbb{R}^{n}$ such that $Q_{i}=\operatorname{conv}\left(V_{i}\right)$ and $C_{i}=\operatorname{cone}\left(R_{i}\right)$. Then it is straightforward to show that $Q=\operatorname{conv}\left(\cup_{i=1}^{k} V_{i}\right)$ and $C=\operatorname{cone}\left(\cup_{i=1}^{k} R_{i}\right)$, thus $Q$ is a polytope and $C$ a finitely generated cone.

We show $\overline{\operatorname{conv}}\left(\cup_{i=1}^{k} P_{i}\right) \subseteq Q+C$.

We just need to show $\operatorname{conv}\left(\cup_{i=1}^{k} P_{i}\right) \subseteq Q+C$, because $Q+C$ is a polyhedron, and so it is closed. Let $x \in \operatorname{conv}\left(\cup_{i=1}^{k} P_{i}\right)$. Then $x$ is a convex combination of a finite number of points in $\cup_{i=1}^{k} P_{i}$. Since $P_{i}$ is convex, we can write $x$ as a convex combination of points $z^{i} \in P_{i}$, say $x=\sum_{i=1}^{k} y_{i} z^{i}$ where $y_{i} \geq 0$ for $i=1, \ldots, k$ and $\sum_{i=1}^{k} y_{i}=1$. Since $P_{i}=Q_{i}+C_{i}$, then $z^{i}=w^{i}+x^{i}$ where $w^{i} \in Q_{i}, x^{i} \in C_{i}$, thus $x=\sum_{i=1}^{k} y_{i} w^{i}+\sum_{i=1}^{k} y_{i} x^{i}$, so $x \in Q+C$ since $\sum_{i=1}^{k} y_{i} w^{i} \in Q$ and $\sum_{i=1}^{k} y_{i} x^{i} \in C$.

We show $Q+C \subseteq \overline{\operatorname{conv}}\left(\cup_{i=1}^{k} P_{i}\right)$.

Let $x \in Q+C$. Then $x=\sum_{i=1}^{k} y_{i} w^{i}+\sum_{i=1}^{k} x^{i}$ where $w^{i} \in Q_{i}, y_{i} \geq 0$, $x^{i} \in C_{i}$ for $i=1, \ldots, k$, and $\sum_{i=1}^{k} y_{i}=1$. 
Define $I:=\left\{i: y_{i}>0\right\}$ and consider the point

$$
x^{\epsilon}:=\sum_{i \in I}\left(y_{i}-\frac{k}{|I|} \epsilon\right) w^{i}+\sum_{i=1}^{k} \epsilon\left(w^{i}+\frac{1}{\epsilon} x^{i}\right)
$$

for $\epsilon>0$ small enough so that $y_{i}-\frac{k}{|I|} \epsilon \geq 0$ for all $i \in I$.

Notice that $x^{\epsilon} \in \operatorname{conv}\left(\cup_{i=1}^{k} P_{i}\right)$ since $\sum_{i \in I}\left(y_{i}-\frac{k}{|I|} \epsilon\right)+\sum_{i=1}^{k} \epsilon=1$ and $w^{i}+\frac{1}{\epsilon} x^{i} \in P_{i}$.

Since $\lim _{\epsilon \rightarrow 0^{+}} x^{\epsilon}=x, x \in \overline{\operatorname{conv}}\left(\cup_{i=1}^{k} P_{i}\right)$.

Corollary 3.2. If $P_{1}, \ldots, P_{k}$ are polyhedra with identical recession cones, then $\operatorname{conv}\left(\cup_{i=1}^{k} P_{i}\right)$ is a polyhedron.

Proof. We leave it as an exercise to the reader to check how the last part of the proof of Theorem 3.1 simplifies to show $Q+C \subseteq \operatorname{conv}\left(\cup_{i=1}^{k} P_{i}\right)$.

Although $\overline{\operatorname{conv}}\left(\cup_{i=1}^{k} P_{i}\right)$ may have exponentially many facets, Balas [2], [3] proved that it is the projection of a higher-dimensional polyhedron $Y$ with a polynomial size representation:

$$
Y:=\left\{\begin{aligned}
A_{i} x^{i} & \leq b^{i} y_{i} \\
\sum x^{i} & =x \\
\sum y_{i} & =1 \\
y_{i} & \geq 0 \text { for } i=1, \ldots, k .
\end{aligned}\right.
$$

In this formulation, $x^{i}$ is a vector in $\mathbb{R}^{n}$ and $y_{i}$ is a scalar, for $i=1, \ldots, k$. The vector $x \in \mathbb{R}^{n}$ corresponds to the original space onto which $Y$ is projected. Thus, the polyhedron $Y$ is defined in $\mathbb{R}^{k n+n+k}$. A formulation with a polynomial number of variables and constraints is said to be compact. The gist of Balas's result is that $\overline{\operatorname{conv}}\left(\cup_{i=1}^{k} P_{i}\right)$ has a compact representation with respect to the systems $A_{i} x \leq b^{i}, i=1, \ldots, k$. This fact will be fundamental in the development of this survey.

Since it is convenient to consider also the case where some of the systems $A_{i} x \leq b^{i}$ are infeasible, we need a condition that is typically satisfied in the context of integer programming.

Given the polyhedra $P_{i}:=\left\{x \in \mathbb{R}^{n}: A_{i} x \leq b^{i}\right\}, i=1, \ldots, k$, let $C_{i}:=\left\{x \in \mathbb{R}^{n}: A_{i} x \leq 0\right\}$. So $C_{i}$ is the recession cone of $P_{i}$ when $P_{i} \neq \emptyset$.

Cone Condition: If $\cup P_{i} \neq \emptyset$, then $C_{j} \subseteq \operatorname{conv}\left(\cup_{i: P_{i} \neq \emptyset} C_{i}\right)$ for $j=1, \ldots, k$. 
By Minkowski-Weil's Theorem 2.14, if $P_{i} \neq \emptyset$ then $P_{i}=Q_{i}+C_{i}$ for some polytope $Q_{i}$. If we let $Q_{i}=\emptyset$ whenever $P_{i}=\emptyset$, then $P_{i}=Q_{i}+C_{i}$, $i=1 \ldots, k$.

Theorem 3.3. (Balas [2, 3]) Consider $k$ polyhedra $P_{i}:=\left\{x \in \mathbb{R}^{n}: A_{i} x \leq\right.$ $\left.b^{i}\right\}$ and let $Y$ be the polyhedron defined in (11). Then

$$
\operatorname{proj}_{x} Y=Q+C \text {. }
$$

where $Q=\operatorname{conv}\left(\cup_{i=1}^{k} Q_{i}\right)$ and $C=\operatorname{conv}\left(\cup_{i=1}^{k} C_{i}\right)$.

Furthermore, if the Cone Condition is satisfied, then

$$
\operatorname{proj}_{x} Y=\overline{\operatorname{conv}}\left(\cup_{i=1}^{k} P_{i}\right) .
$$

Proof. Notice that, if the Cone Condition is satisfied, then $C=\operatorname{conv}\left(\cup_{i: P_{i} \neq \emptyset} C_{i}\right)$, therefore by Theorem $3.1 Q+C=\overline{\operatorname{conv}}\left(\cup_{i=1}^{k} P_{i}\right)$. Thus, if $\operatorname{proj}_{x} Y=Q+C$, then $\operatorname{proj}_{x} Y=\overline{\operatorname{conv}}\left(\cup_{i=1}^{k} P_{i}\right)$. So we only need to $\operatorname{show}_{\operatorname{proj}_{x}} Y=Q+C$.

(a) $Q+C \subseteq \operatorname{proj}_{x} Y$.

The result holds trivially when $\cup_{i=1}^{k} P_{i}=\emptyset$, so we assume $\cup_{i=1}^{k} P_{i} \neq \emptyset$. Without loss of generality, $P_{1}, \ldots, P_{h}$ are nonempty and $P_{h+1}, \ldots, P_{k}$ are empty, where $1 \leq h \leq k$.

Let $x \in Q+C$. Then there exist $w^{i} \in Q^{i}, i=1, \ldots, h$, and $z^{i} \in C_{i}$, $i=1, \ldots, k$, such that $x=\sum_{i=1}^{h} y_{i} w^{i}+\sum_{i=1}^{k} z^{i}$, where $y_{i} \geq 0$ for $i=1, \ldots, h$ and $\sum_{i=1}^{h} y_{i}=1$. Let $x^{i}=y_{i} w^{i}+z^{i}$ for $i=1, \ldots, h$ and $y_{i}=0, x^{i}=z^{i}$ for $i=h+1, \ldots, k$. Then $A_{i} x^{i} \leq b^{i} y_{i}$ for $i=1, \ldots k$ and $x=\sum_{i=1}^{k} x^{i}$. This shows that $x \in \operatorname{proj}_{x} Y$ and therefore (a) holds.

(b) $\operatorname{proj}_{x} Y \subseteq Q+C$.

The result holds if $Y=\emptyset$, so we assume $Y \neq \emptyset$. Let $x \in \operatorname{proj}_{x} Y$. By the definition of projection, there exist $x^{1}, \ldots, x^{k}, y$ such that $x=\sum_{i=1}^{k} x^{i}$ where $A x^{i} \leq b^{i} y_{i}, \sum y_{i}=1, y \geq 0$. Let $I:=\left\{i: y_{i}>0\right\}$.

For $i \in I$, let $z^{i}:=\frac{x^{i}}{y_{i}}$. Then $z^{i} \in P_{i}$. Since $P_{i}=Q_{i}+C_{i}$, we can write $z^{i}=w^{i}+y^{i} r^{i}$ where $w^{i} \in Q_{i}$ and $r^{i} \in C_{i}$.

For $i \notin I$, we have $A_{i} x^{i} \leq 0$, that is $x^{i} \in C_{i}$. Let $r^{i}=x^{i}$ if $i \notin I$. Then

$$
x=\sum_{i \in I} y_{i} z^{i}+\sum_{i \notin I} x^{i}=\underbrace{\sum_{i \in I} y_{i} w^{i}}_{\in Q}+\underbrace{\sum_{i=1}^{k} r^{i}}_{\in C} \in Q+C .
$$


Remark 3.4. The Cone Condition assumption in Theorem 3.3 is necessary as shown by the following example (see Figure 5): $P_{1}:=\left\{x \in \mathbb{R}^{2}: 0 \leq x \leq\right.$ $1\}$ and $P_{2}:=\left\{x \in \mathbb{R}^{2}: x_{1} \leq 0, x_{1} \geq 1\right\}$. Note that $P_{2}=\emptyset$ and $C_{2}=\{x \in$ $\left.\mathbb{R}^{2}: x_{1}=0\right\}$. So in this case $\operatorname{proj}_{x} Y=P_{1}+C_{2}=\left\{x \in \mathbb{R}^{2}: 0 \leq x_{1} \leq 1\right\}$, which is different from $\overline{\operatorname{conv}}\left(P_{1} \cup P_{2}\right)=P_{1}$.



Figure 5: $\overline{\operatorname{conv}}\left(P_{1} \cup P_{2}\right) \neq \operatorname{proj}_{x} Y$

Remark 3.5. The Cone Condition assumption in Theorem 3.3 is automatically satisfied if

(i) $C_{i}=\{0\}$ whenever $P_{i}=\emptyset$, or

(ii) all the cones $C_{i}$ are identical.

For example (i) holds when all the $P_{i}$ s are nonempty, or when $C_{i}=\{0\}$ for all $i$.

\section{Split disjunctions}

Let $P:=\left\{(x, y) \in \mathbb{R}^{n} \times \mathbb{R}^{p}: A x+G y \leq b\right\}$ and let $S:=P \cap\left(\mathbb{Z}^{n} \times \mathbb{R}^{p}\right)$. For $\pi \in \mathbb{Z}^{n}$ and $\pi_{0} \in \mathbb{Z}$, define

$$
\begin{aligned}
& \Pi_{1}=P \cap\left\{(x, y): \pi x \leq \pi_{0}\right\} \\
& \Pi_{2}=P \cap\left\{(x, y): \pi x \geq \pi_{0}+1\right\} .
\end{aligned}
$$

Clearly $S \subseteq \Pi_{1} \cup \Pi_{2}$ and therefore $\operatorname{conv}(S) \subseteq \operatorname{conv}\left(\Pi_{1} \cup \Pi_{2}\right)$. We call this latter set $P^{\left(\pi, \pi_{0}\right)}$.

Theorem 4.1. $P^{\left(\pi, \pi_{0}\right)}$ is a polyhedron. 
Proof. It follows from Theorem 3.1 that $\overline{P^{\left(\pi, \pi_{0}\right)}}$ is a polyhedron, thus we only need to show that $P^{\left(\pi, \pi_{0}\right)}$ is closed. This is obvious if at least one of $\Pi_{1}$ and $\Pi_{2}$ is empty, so we assume $\Pi_{1}, \Pi_{2} \neq \emptyset$. Because $\Pi_{1} \cup \Pi_{2} \subset P^{\left(\pi, \pi_{0}\right)} \subseteq P$, $\Pi_{1}=P^{\left(\pi, \pi_{0}\right)} \cap\left\{(x, y): \pi x \leq \pi_{0}\right\}$ and $\Pi_{2}=P^{\left(\pi, \pi_{0}\right)} \cap\left\{(x, y): \pi x \geq \pi_{0}+1\right\}$. Thus $P^{\left(\pi, \pi_{0}\right)}=\Pi_{1} \cup \Pi_{2} \cup \Pi$, where $\Pi=P^{\left(\pi, \pi_{0}\right)} \cap\left\{(x, y): \pi_{0} \leq \pi x \leq \pi_{0}+1\right\}$. By definition, $\Pi_{1}$ and $\Pi_{2}$ are polyhedra, so they are closed. We show that $\Pi$ is a polyhedron, thus $P^{\left(\pi, \pi_{0}\right)}$ is closed because it is the union of a finite number of closed sets.

Let $P_{1}:=\Pi_{1} \cap\left\{(x, y): \pi x=\pi_{0}\right\}$ and $P_{2}:=\Pi_{2} \cap\left\{(x, y): \pi x=\pi_{0}+1\right\}$. Notice that $P_{1}$ and $P_{2}$ have the same recession cone $C:=\{(x, y): A x+G y \leq$ $0, \pi x=0\}$, thus, by Corollary $3.2, \operatorname{conv}\left(P_{1} \cup P_{2}\right)$ is a polyhedron.

We show that $\Pi=\operatorname{conv}\left(P_{1} \cup P_{2}\right)$, thus showing that $\Pi$ is a polyhedron.

The inclusion $\Pi \supseteq \operatorname{conv}\left(P_{1} \cup P_{2}\right)$ comes from the definition. We prove $\Pi \subseteq \operatorname{conv}\left(P_{1} \cup P_{2}\right)$. If $(\bar{x}, \bar{y}) \in \Pi$, then there exist $\left(x^{\prime}, y^{\prime}\right) \in \Pi_{1},\left(x^{\prime \prime}, y^{\prime \prime}\right) \in \Pi_{2}$ such that $(\bar{x}, \bar{y})$ is contained in the line segment $L$ joining $\left(x^{\prime}, y^{\prime}\right)$ and $\left(x^{\prime \prime}, y^{\prime \prime}\right)$. Since $\pi_{0} \leq \pi \bar{x} \leq \pi_{0}+1, L$ intersect $\left\{(x, y): \pi x=\pi_{0}\right\}$ in a point $\left(\bar{x}^{\prime}, \bar{y}^{\prime}\right) \in$ $\Pi_{1}$, and $\left\{(x, y): \pi x=\pi_{0}+1\right\}$ in a point $\left(\bar{x}^{\prime \prime}, \bar{y}^{\prime \prime}\right) \in \Pi_{2}$. Furthermore, $(\bar{x}, \bar{y})$ is contained in the line segment joining $\left(\bar{x}^{\prime}, \bar{y}^{\prime}\right)$ and $\left(\bar{x}^{\prime \prime}, \bar{y}^{\prime \prime}\right)$. Thus $(\bar{x}, \bar{y}) \in \operatorname{conv}\left(P_{1} \cup P_{2}\right)$.

Lemma 4.2. The polyhedra $\Pi_{1}$ and $\Pi_{2}$ satisfy the Cone Condition of Theorem 3.3 .

Proof. The conditions of Theorem 3.3 trivially hold when $\Pi_{1}$ and $\Pi_{2}$ are either both empty or both nonempty.

Assume now that $\Pi_{1}=\emptyset$ and $\Pi_{2} \neq \emptyset$. For the conditions of Theorem 3.3 to hold, we need to show that $C_{1} \subseteq C_{2}$ where $C_{1}:=\{(x, y): A x+G y \leq$ $0, \pi x \leq 0\}$ and $C_{2}:=\{(x, y): A x+G y \leq 0, \pi x \geq 0\}$.

We claim that $C_{1}=\{(x, y): A x+G y \leq 0, \pi x=0\}$. Suppose not. Then there exists $(\bar{x}, \bar{y})$ such that $A \bar{x}+G \bar{y} \leq 0$ and $\pi \bar{x}<0$. Since $\Pi_{2} \neq \emptyset$, there exists $(\hat{x}, \hat{y})$ such that $A \hat{x}+G \hat{y} \leq b$. Now consider the point $\left(x^{\lambda}, y^{\lambda}\right)=$ $(\hat{x}, \hat{y})+\lambda(\bar{x}, \bar{y})$. We have $A x^{\lambda}+G y^{\lambda} \leq b$ and, for $\lambda \geq \frac{\pi \hat{x}-\pi_{0}}{|\pi \bar{x}|}$, we have $\pi x^{\lambda} \leq \pi_{0}$. But then $\left(x^{\lambda}, y^{\lambda}\right)$ is in $\Pi_{1}$, contradicting the assumption that $\Pi_{1}=\emptyset$. This proves the claim.

The claim implies $C_{1} \subseteq C_{2}$.

Thus, by Theorem $3.3, P^{\left(\pi, \pi_{0}\right)}$ has the following extended formulation, 
with additional vectors of variables $\left(x^{1}, y^{1}\right),\left(x^{2}, y^{2}\right)$ and variable $\lambda$.

$$
\begin{array}{cl}
A x^{1}+G y^{1} & \leq \lambda b \\
\pi x^{1} & \leq \lambda \pi_{0} \\
A x^{2}+G y^{2} & \leq(1-\lambda) b \\
\pi x^{2} & \geq(1-\lambda)\left(\pi_{0}+1\right) \\
x^{1}+x^{2} & =x \\
y^{1}+y^{2} & =y \\
0 \leq \lambda & \leq \mathbf{1} .
\end{array}
$$

If the system $A x+G y \leq b$ has $m$ constraints, the extended formulation (13) has $2 m+n+p+4$ constraints. By contrast, a formulation of $P^{\left(\pi, \pi_{0}\right)}$ in the original space $(x, y)$ may be considerably more complicated, as $P^{\left(\pi, \pi_{0}\right)}$ may have a large number of facets (Recall from Section 2.7 that any description of a polyhedron must have at least one inequality for each facet).



Figure 6: A split inequality

A split is a disjunction $\pi x \leq \pi_{0}$ or $\pi x \geq \pi_{0}+1$ where $\pi \in \mathbb{Z}^{n}$ and $\pi_{0} \in \mathbb{Z}$. We will also say that $\left(\pi, \pi_{0}\right)$ defines a split. An inequality $c x+h y \leq c_{0}$ is called a split inequality [17] if it is valid for some polyhedron $P^{\left(\pi, \pi_{0}\right)}$ where $\left(\pi, \pi_{0}\right) \in \mathbb{Z}^{n} \times \mathbb{Z}$ defines a split (see Figure 6).

The split closure $P^{\text {Split }}$ of $P$ is

$$
\bigcap_{\left(\pi, \pi_{0}\right) \in \mathbb{Z}^{n} \times \mathbb{Z}} P^{\left(\pi, \pi_{0}\right)} .
$$

Clearly $S \subseteq P^{S p l i t} \subseteq P$. In general $P^{S p l i t}$ provides a tighter relaxation of $S$ than $P$. Although each of the sets $P^{\left(\pi, \pi_{0}\right)}$ is a polyhedron, it is not clear 
however that $P^{\text {Split }}$ is a polyhedral set, for it is the intersection of infinitely many of them. This will be discussed in Section 6 .

\subsection{One-side splits, Chvátal inequalities}

Given a polyhedron $P:=\left\{(x, y) \in \mathbb{R}^{n} \times \mathbb{R}^{p}: A x+G y \leq b\right\}$, we consider the mixed integer set $S=P \cap\left(\mathbb{Z}^{n} \times \mathbb{R}^{p}\right)$. Let $\pi \in \mathbb{Z}^{n}$ and $z:=\max \{\pi x$ : $(x, y) \in P\}$. A split defined by $\left(\pi, \pi_{0}\right) \in \mathbb{Z}^{n} \times \mathbb{Z}$ is a one-side split for $P$ if

$$
\pi_{0} \leq z<\pi_{0}+1 \text {. }
$$

This is equivalent to:

$$
\Pi_{1} \subseteq P \text { and } \Pi_{2}=\emptyset
$$

where $\Pi_{1}$ and $\Pi_{2}$ are the polyhedra defined in (12). Note that, if $\left(\pi, \pi_{0}\right)$ defines a one-side split, then the polyhedron $P^{\left(\pi, \pi_{0}\right)}$ can be easily described in its original space, for $P^{\left(\pi, \pi_{0}\right)}=\Pi_{1}=\left\{(x, y): A x+G y \leq b, \pi x \leq \pi_{0}\right\}$. The inequality $\pi x \leq \pi_{0}$ is valid for $S$. It is called a Chvátal inequality.

The Chvátal closure $P^{C h}$ of $P$ is

$$
\bigcap_{\left(\pi, \pi_{0}\right) \in \mathbb{Z}^{n} \times \mathbb{Z} \text { defines a one-side split }} P^{\left(\pi, \pi_{0}\right)} .
$$

Equivalently, $P^{C h}$ is the set of vectors $(x, y)$ that satisfy the system $A x+$ $G y \leq b$ and all the Chvátal inequalities. Note that $S \subseteq P^{S p l i t} \subseteq P^{C h} \subseteq P$.

Notice that inequality $\pi x \leq \pi_{0}$ satisfies (15) if and only if $\lfloor z\rfloor=\pi_{0}$. Since $\pi$ is an integral vector and $\pi x \leq z$ is a valid inequality for $P$, by Theorem 2.21, $\pi x \leq \pi_{0}$ is a Chvátal inequality if and only if the system:

$$
u \geq 0, u A=\pi \in \mathbb{Z}^{n}, u G=0, u b \leq z
$$

is feasible. By Theorem 2.16, condition $u \geq 0, u G=0$ shows that $\pi x \leq z$ is valid for the projection $\operatorname{proj}_{x}(P)$ of $P$ onto the $x$-space.

We can assume that $u$ is chosen so that the coefficients of $\pi$ are relatively prime: If not, let $m$ be the G.C.D. of the components of $\pi$ : The inequality $\frac{\pi}{m} x \leq\left\lfloor\frac{\pi_{0}}{m}\right\rfloor$ is a Chvátal inequality that dominates $\pi x \leq \pi_{0}$. The equation $\pi x=\pi_{0}=\lfloor z\rfloor$ admits infinitely many integral solutions, while the equation $\pi x=\alpha$, has obviously no integral solution for $\lfloor z\rfloor<\alpha \leq z$.

Therefore the Chvátal closure is obtained by taking any rational inequality $\pi x \leq z$ that is valid for the projection $\operatorname{proj}_{x}(P)$ of $P$ (which is generated 


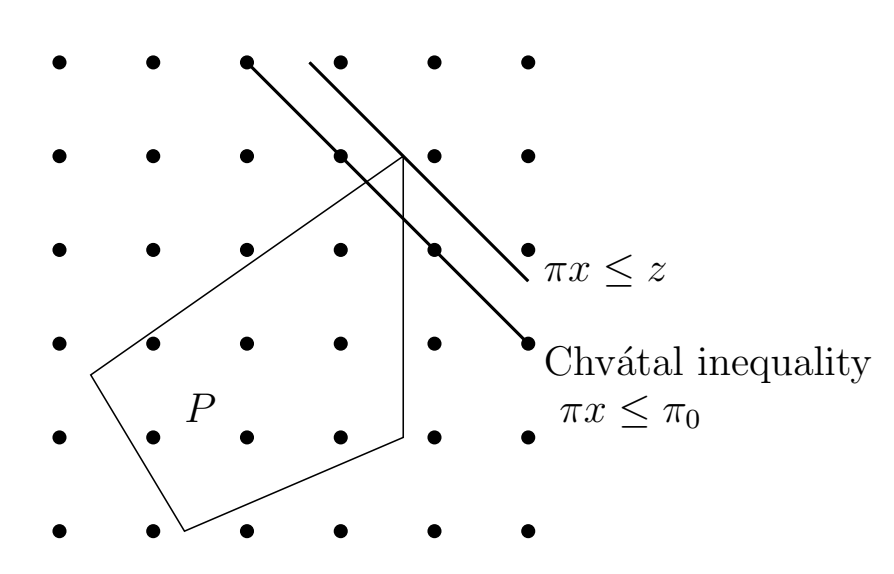

Figure 7: Chvátal inequality

without loss of generality by a vector $u$ such that $u \geq 0, u A=\pi \in \mathbb{Z}^{n}, u G=$ 0 such that the coefficients of $\pi$ are relatively prime) and then tightening the inequality by decreasing the right-hand side until an integral point (and hence infinitely many integral points) are encountered. See Figure 7.

\section{Gomory's mixed-integer inequalities}

We consider a polyhedron $P:=\left\{(x, y) \in \mathbb{R}_{+}^{n} \times \mathbb{R}_{+}^{p}: A x+G y \leq b\right\}$ and the set $S:=P \cap\left(\mathbb{Z}_{+}^{n} \times \mathbb{R}_{+}^{p}\right)$. Note that, here, $P$ is defined by a system of inequalities together with nonnegativity constraints on the variables, and this is important in this section. By standard linear algebra, any system of linear inequalities can be converted into a system of the above type (variables that are unrestricted in sign can be replaced by the difference of two nonnegative variables, etc).

We consider the following equality form of the system defining $P$ :

$$
A x+G y+I s=b, x, y, s \geq 0 .
$$

For $\lambda \in \mathbb{Q}^{m}$, we consider the equation $\lambda A x+\lambda G y+\lambda I s=\lambda b$, which we denote by

$$
\sum_{j=1}^{n} a_{j}^{\lambda} x_{j}+\sum_{j=1}^{p} g_{j}^{\lambda} y_{j}+\sum_{i=1}^{m} \lambda_{i} s_{i}=\beta^{\lambda} .
$$

Let $f_{0}=\beta^{\lambda}-\left\lfloor\beta^{\lambda}\right\rfloor$ and $f_{j}=a_{j}^{\lambda}-\left\lfloor a_{j}^{\lambda}\right\rfloor$. We consider the following 
Gomory mixed-integer (GMI) inequality [32] :

$$
\sum_{j=1}^{n}\left(\left\lfloor a_{j}^{\lambda}\right\rfloor+\frac{\left(f_{j}-f_{0}\right)^{+}}{1-f_{0}}\right) x_{j}+\frac{1}{1-f_{0}}\left(\sum_{j: g_{j}^{\lambda}<0} g_{j}^{\lambda} y_{j}+\sum_{i: \lambda_{i}<0} \lambda_{i} s_{i}\right) \leq\left\lfloor\beta^{\lambda}\right\rfloor
$$

where $\left(f_{j}-f_{0}\right)^{+}=\max \left\{f_{j}-f_{0}, 0\right\}$. By substituting $s=b-(A x+G y)$ we get an inequality in the $(x, y)$-space.

We denote by $\left(\pi^{\lambda}, \pi_{0}^{\lambda}\right)$ the vector in $\mathbb{Z}^{n} \times \mathbb{Z}$ defining the following split:

$$
\begin{aligned}
& \text { Either } \sum_{f_{j} \leq f_{0}}\left\lfloor a_{j}^{\lambda}\right\rfloor x_{j}+\sum_{f_{j}>f_{0}}\left\lceil a_{j}^{\lambda}\right\rceil x_{j} \leq\left\lfloor\beta^{\lambda}\right\rfloor \\
& \text { or } \sum_{f_{j} \leq f_{0}}\left\lfloor a_{j}^{\lambda}\right\rfloor x_{j}+\sum_{f_{j}>f_{0}}\left\lceil a_{j}^{\lambda}\right\rceil x_{j} \geq\left\lfloor\beta^{\lambda}\right\rfloor+1 ;
\end{aligned}
$$

where $(21)$ is $\pi^{\lambda} x \leq \pi_{0}^{\lambda}$ while $(22)$ is $\pi^{\lambda} x \geq \pi_{0}^{\lambda}+1$.

Lemma 5.1. Inequality (20) is a split inequality, valid for $P^{\left(\pi^{\lambda}, \pi_{0}^{\lambda}\right)}$.

Proof. Consider the sets

$$
\Pi_{1}=P \cap\left\{(x, y): \pi^{\lambda} x \leq \pi_{0}^{\lambda}\right\}, \quad \Pi_{2}=P \cap\left\{(x, y): \pi^{\lambda} x \geq \pi_{0}^{\lambda}+1\right\} .
$$

To prove that inequality (20) is a split inequality, we will show that it is valid for $\Pi_{1} \cup \Pi_{2}$.

Since the constraints $x \geq 0, y \geq 0, s \geq 0$ are valid for $\Pi_{1}$ and $\Pi_{2}$, it is enough to show that each of $\Pi_{1}$ and $\Pi_{2}$ admits a valid inequality $a x+g y+$ $l s \leq\left\lfloor\beta^{\lambda}\right\rfloor$ whose coefficients are greater than or equal to the corresponding coefficients in inequality (20). (This is where the nonnegativity plays a role).

Since inequality $\pi^{\lambda} x \leq \pi_{0}^{\lambda}$ has this property, inequality (20) is valid for $\Pi_{1}$. Inequality (20) is valid for $\Pi_{2}$ since it is implied by the inequality

$$
\frac{1}{1-f_{0}}(19)-\frac{f_{0}}{1-f_{0}}(22) \text {. }
$$

A consequence of the above lemma is that the GMI inequalities (20) are valid for $S$.

Lemma 5.2. Chvátal inequalities are GMI inequalities. 
Proof. For $\lambda \geq 0$ such that $\lambda A \in \mathbb{Z}^{n}$ and $\lambda G=0$ the Chvátal inequality (17) coincides with the inequality given by the formula (20).

The Gomory mixed integer closure $P^{G M I}$ of $P$ is the set of points in $P$ that satisfy all the GMI inequalities (20). It follows from Lemmas 5.1 and 5.2 that $P^{S p l i t} \subseteq P^{G M I} \subseteq P^{C h}$. In light of the particular derivation of the GMI inequalities, it may appear that the first containment can be strict. This is not the case: In the next section we show that $P^{S p l i t}$ coincides with $P^{G M I}$.

\subsection{Equivalence between split closure and Gomory mixed integer closure}

In this section we will need the following.

Lemma 5.3. Let $P:=\left\{x \in \mathbb{R}^{n}: A x \leq b\right\}$ be a polyhedron and let $\Pi:=$ $P \cap\left\{x: \pi x \leq \pi_{0}\right\}$. If $\Pi \neq \emptyset$ and $\alpha x \leq \beta$ is a valid inequality for $\Pi$, then there exists a scalar $\lambda \in \mathbb{R}_{+}$such that

$$
\alpha x-\lambda\left(\pi x-\pi_{0}\right) \leq \beta
$$

is valid for $P$.

Proof. By Theorem 2.21, since $\Pi \neq \emptyset$, there exist $u \geq 0, \lambda \geq 0$ such that

$$
\alpha=u A+\lambda \pi \quad \text { and } \quad \beta \geq u b+\lambda \pi_{0} .
$$

Since $u A x \leq u b$ is valid for $P$, so is $u A x \leq \beta-\lambda \pi_{0}$. Since $u A x=\alpha x-\lambda \pi x$, the inequality $\alpha x-\lambda\left(\pi x-\pi_{0}\right) \leq \beta$ is valid for $P$.

Remark 5.4. The assumption $\Pi \neq \emptyset$ is necessary in Lemma 5.3, as shown by the following example: $P:=\left\{x \in \mathbb{R}^{2}: x_{1} \geq 0, x_{2} \geq 0\right\}$ and $\Pi:=P \cap\{x$ : $\left.x_{2} \leq-1\right\}$. Thus $\Pi$ is empty. The inequality $x_{1} \leq 1$ is valid for $\Pi$ but there is no scalar $\lambda$ such that $x_{1}-\lambda\left(x_{2}+1\right) \leq 1$ is valid for $P$.

Theorem 5.5. (Nemhauser and Wolsey [40]) Let $P:=\left\{(x, y) \in \mathbb{R}_{+}^{n} \times \mathbb{R}_{+}^{p}\right.$ : $A x+G y \leq b\}$ be a polyhedron and let $S:=P \cap\left(\mathbb{Z}_{+}^{n} \times \mathbb{R}_{+}^{p}\right)$. Then $P^{\text {Split }}$ coincides with $P^{G M I}$.

Proof. (Cornuéjols and Li [18]) Lemma 5.1 shows that $P^{S p l i t} \subseteq P^{G M I}$. To prove the reverse inclusion, we show that every split inequality is a GMI inequality. 


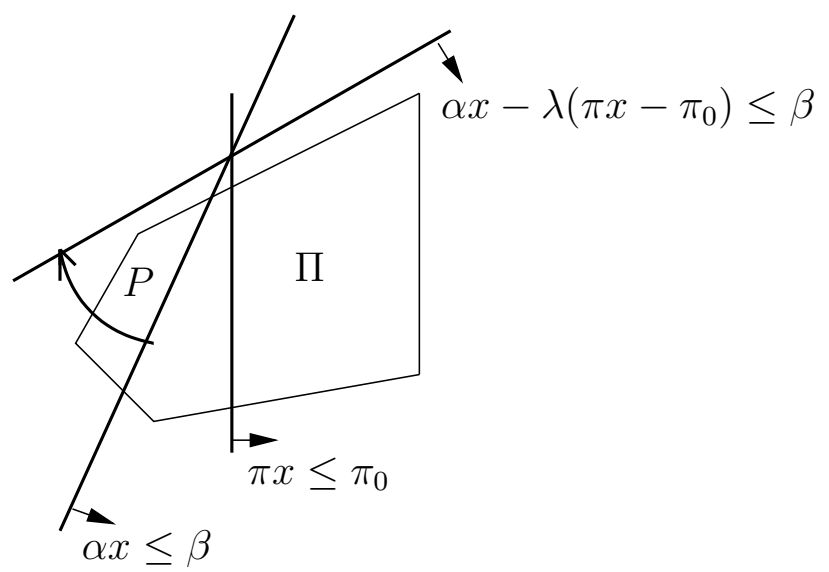

Figure 8: Illustration of Lemma 5.3

We assume that the constraints $x \geq 0$ and $y \geq 0$ are part of the system $A x+G y \leq b$ that defines $P$. Let $c x+h y \leq c_{0}$ be a split inequality. Let $\left(\pi, \pi_{0}\right) \in \mathbb{Z}^{n} \times \mathbb{Z}$ define the split disjunction used in deriving this inequality and let $\Pi_{1}, \Pi_{2}$ be the corresponding intersections with $P$ as defined in (12).

First assume that one of $\Pi_{1}, \Pi_{2}$ is empty. Then the inequality $c x+h y \leq$ $c_{0}$ is a Chvátal inequality and by Lemma 5.2 it is also a GMI inequality.

We now assume that both $\Pi_{1}, \Pi_{2}$ are nonempty. By Lemma 5.3, there exist $\alpha, \beta \in \mathbb{R}_{+}$such that

$$
\begin{aligned}
& c x+h y-\alpha\left(\pi x-\pi_{0}\right) \leq c_{0} \text { and } \\
& c x+h y+\beta\left(\pi x-\left(\pi_{0}+1\right)\right) \leq c_{0}
\end{aligned}
$$

are both valid for $P$. We can assume $\alpha>0$ and $\beta>0$ since, otherwise, $c x+h y \leq c_{0}$ is valid for $P$ and therefore also for $P^{G M I}$. We now introduce slack variables $s_{1}$ and $s_{2}$ in (23) and (24) respectively and subtract (23) from (24). We obtain

$$
(\alpha+\beta) \pi x+s_{2}-s_{1}=(\alpha+\beta) \pi_{0}+\beta .
$$

Dividing by $\alpha+\beta$ we get

$$
\pi x+\frac{s_{2}}{\alpha+\beta}-\frac{s_{1}}{\alpha+\beta}=\pi_{0}+\frac{\beta}{\alpha+\beta} .
$$

We now derive the GMI inequality associated with equation (25). Note that the fractional part of the right-hand side is $\frac{\beta}{\alpha+\beta}$ and that the continuous 
variable $s_{2}$ has a positive coefficient while $s_{1}$ has a negative coefficient. So the GMI inequality is

$$
\pi x+\frac{1}{\alpha} s_{1} \leq \pi_{0} .
$$

We now use (23) to eliminate $s_{1}$ to get the GMI inequality in the space of the $x, y$ variables. The resulting inequality is $c x+h y \leq c_{0}$ and therefore $c x+h y \leq c_{0}$ is a GMI inequality.

\section{Polyhedrality of closures}

In this section we show that the GMI closure (or equivalently, the split closure) of a rational polyhedron is a rational polyhedron. This result is due to Cook, Kannan and Schrijver [17]. Simpler proofs appear in [1], [22] and [47]. In this section, we follow the approach of Dash, Günlük and Lodi [22]. The idea is to prove that a finite number of splits are sufficient to generate $P^{\text {Split }}$ defined in (14). The result follows, since then $P^{\text {Split }}$ is the intersection of a finite number of polyhedra and therefore is a polyhedron.

For this idea to work, it is fundamental to assume that the polyhedra that we deal with are rational. We first illustrate this in an easy case: The Chvátal closure of a pure integer set.

\subsection{The Chvátal closure of a pure integer set}

We consider here pure integer sets of the type $S:=P \cap \mathbb{Z}^{n}$, where $P:=$ $\left\{x \in \mathbb{R}^{n}: A x \leq b\right\}$ is a rational polyhedron. Therefore we can assume that $A, b$ have integral entries.

In the pure integer case, a Chvátal inequality $\pi x \leq \pi_{0}$ is derived from a vector $u$ satisfying:

$$
u \geq 0, u A=\pi \in \mathbb{Z}^{n},\lfloor u b\rfloor=\pi_{0} .
$$

Lemma 6.1. Let $\left(\pi, \pi_{0}\right) \in \mathbb{Z}^{n+1}$ and $u \in \mathbb{Q}$ satisfying (26) such that $\pi x \leq$ $\pi_{0}$ is not valid for $P$ and not redundant for $P^{C h}$. Then $u<\mathbf{1}$.

Proof. Suppose $u$ does not satisfy $u<\mathbf{1}$. We will show that $\pi x \leq \pi_{0}$ is the sum of a Chvátal inequality $\pi^{1} x \leq \pi_{0}^{1}$ and an inequality $\pi^{2} x \leq \overline{\pi_{0}^{2}}$ valid for $P$ such that $\pi^{2} \neq 0$. This contradicts the assumption that $\pi x \leq \pi_{0}$ is not valid for $P$ and not redundant for $P^{C h}$.

Let $f=u-\lfloor u\rfloor$, and let $\pi^{1}=f A, \pi_{0}^{1}=\lfloor f b\rfloor, \pi^{2}=\lfloor u\rfloor A, \pi_{0}^{2}=\lfloor\lfloor u\rfloor b\rfloor$. Since $A$ is an integral matrix, $\pi^{2}$ is an integral vector. Since $\pi$ is an integral vector, $\pi^{1}=\pi-\pi^{2}$ is integral as well. 
Since $b$ is integral, $\pi_{0}^{2}=\lfloor u\rfloor b$, therefore $\pi^{2} x \leq \pi_{0}^{2}$ is valid for $P$. Furthermore, $\pi x \leq \pi_{0}$ is the sum of $\pi^{1} x \leq \pi_{0}^{1}$ and $\pi^{2} x \leq \pi_{0}^{2}$. Since $\pi x \leq \pi_{0}$ is not valid for $P$, then $f \neq 0$. Since $u$ does not satisfy $u<\mathbf{1}$, then $\lfloor u\rfloor \neq 0$, thus $\pi^{2} \neq 0$.

Theorem 6.2. (Chvátal [13]) $P^{C h}$ is a rational polyhedron.

Proof. By Lemma 6.1, any irredundant valid inequality for $P^{C h}$ that is not valid for $P$ is of the form $(u A) x \leq\lfloor u b\rfloor$ for some $u$ satisfying $u A \in \mathbb{Z}^{n}, 0 \leq$ $u<\mathbf{1}$. Since $\left\{u A \in \mathbb{R}^{n}: 0 \leq u<\mathbf{1}\right\}$ is bounded, $\left\{u A \in \mathbb{Z}^{n}: 0 \leq u<\mathbf{1}\right\}$ is finite. Thus there is only a finite number of such inequalities, hence $P^{C h}$ is a polyhedron.

\subsection{The split closure of a mixed integer set}

This is more tricky, uses the fact that $P^{S p l i t}=P^{G M I}$ (Theorem 5.5) but the idea is the same.

Throughout this section, $P:=\left\{(x, y) \in \mathbb{R}_{+}^{n} \times \mathbb{R}_{+}^{p}: A x+G y \leq b\right\}$ is a rational polyhedron, and $S:=P \cap\left(\mathbb{Z}_{+}^{n} \times \mathbb{R}_{+}^{p}\right)$. We will also assume that $A$, $G, b$ are integral. We let $s=b-(A x+G y)$ be the slacks of $A x+G y \leq b$. Recall from Section 5 that a vector $\lambda \in \mathbb{Q}^{m}$ yields the GMI inequality:

$$
\sum_{j=1}^{n}\left(\left\lfloor a_{j}^{\lambda}\right\rfloor+\frac{\left(f_{j}-f_{0}\right)^{+}}{1-f_{0}}\right) x_{j}+\frac{1}{1-f_{0}} \sum_{j: g_{j}^{\lambda}<0} g_{j}^{\lambda} y_{j}+\sum_{i: \lambda_{i}<0}+\lambda_{i} s_{i} \leq\left\lfloor\beta^{\lambda}\right\rfloor
$$

which is valid for $S$. We denote it by $G M(\lambda)$.

Given $\lambda \in \mathbb{Q}^{m}$ and the corresponding GMI inequality $G M(\lambda)$, we consider the following partitions of $M:=\{1, \ldots, m\}$ and $P:=\{1, \ldots, p\}$ :

$$
\begin{array}{cl}
M^{+}=\left\{i \in M: \lambda_{i} \geq 0\right\} & M^{-}=\left\{i \in M: \lambda_{i}<0\right\} \\
P^{+}=\left\{j \in P: g_{j}^{\lambda} \geq 0\right\} & P^{-}=\left\{j \in P: g_{j}^{\lambda}<0\right\}
\end{array}
$$

and the following cone:

$C_{\lambda}=\left\{\mu \in \mathbb{R}^{m}: g_{j}^{\mu} \geq 0, j \in P^{+}, g_{j}^{\mu} \leq 0, j \in P^{-}, \mu_{i} \geq 0, i \in M^{+}, \mu_{i} \leq 0, i \in M^{-}\right\}$

Lemma 6.3. Let $\lambda \in \mathbb{Q}^{m}$. Let $\lambda^{1}, \lambda^{2} \in C_{\lambda}$ such that $\lambda=\lambda^{1}+\lambda^{2}$ and $\lambda^{2} \in \mathbb{Z}^{m} \backslash\{0\}$. Then

$$
G M(\lambda)=G M\left(\lambda^{1}\right)+G M\left(\lambda^{2}\right)
$$

and either $G M(\lambda)$ is valid for $P$ or it is redundant for $P^{G M I}$. 
Proof. Since $\lambda^{2} b$ is an integer, $f_{0}=0$ and $G M\left(\lambda^{2}\right)$ is the following inequality:

$$
\sum_{j=1}^{n} a_{j}^{\lambda^{2}} x_{j}+\sum_{j: g_{j}^{\lambda^{2}}<0} g_{j}^{\lambda^{2}} y_{j}+\sum_{i: \lambda_{i}^{2}<0}+\lambda_{i}^{2} s_{i} \leq \beta^{\lambda^{2}} .
$$

Therefore $G M\left(\lambda^{2}\right)$ is implied by the following inequalities, valid for $P$ :

$$
\sum_{j=1}^{n} a_{j}^{\lambda^{2}} x_{j}+\sum_{j=1}^{p} g_{j}^{\lambda^{2}} y_{j}+\sum_{i=1}^{m} \lambda_{i}^{2} s_{i}=\beta^{\lambda^{2}}, \quad s \geq 0, \quad y \geq 0 .
$$

Hence $G M\left(\lambda^{2}\right)$ is valid for $P$. Moreover, since $A, G, b$ are integral, all coefficients of $G M\left(\lambda^{2}\right)$ are integral.

Since $\lambda^{1}, \lambda^{2} \in C_{\lambda}, g_{j}^{\lambda}<0$ implies $g_{j}^{\lambda^{1}} \leq 0$ and $g_{j}^{\lambda^{2}} \leq 0$; and $\lambda_{i}<0$ implies $\lambda_{i}^{1} \leq 0$ and $\lambda_{i}^{2} \leq 0$. This shows that $G M(\lambda)=G M\left(\lambda^{1}\right)+G M\left(\lambda^{2}\right)$. Thus, since $G M\left(\lambda^{2}\right)$ is valid for $P$, either $G M(\lambda)$ is valid for $P$, or it is redundant for $P^{G M I}$.

Let $\Delta$ be the largest of the absolute values of the determinants of the square submatrices of $G$.

Lemma 6.4. Let $\lambda \in \mathbb{Q}^{m}$ such that $G M(\lambda)$ is not valid for $P$ and it is irredundant for $P^{G M I}$. Then

$$
-m \Delta \leq \lambda_{i} \leq m \Delta, \quad i=1, \ldots, m .
$$

Proof. We will show that if $\lambda$ does not satisfy (28), then there exist $\lambda^{1}, \lambda^{2} \in$ $C_{\lambda}$ such that $\lambda=\lambda^{1}+\lambda^{2}$ and $\lambda^{2} \in \mathbb{Z}^{m} \backslash\{0\}$. This will prove the lemma since then, by Lemma 6.3, either $G M(\lambda)$ is valid for $P$ or it is redundant for $P^{G M I}$, a contradiction.

Assume $\lambda$ violates (28). Let $r^{1}, \ldots, r^{q}$ be a set of vectors generating $C_{\lambda}$. By Remark 2.13, we can choose $r^{1}, \ldots, r^{q}$ integral, and by standard linear algebra we can choose them so that $-\Delta \mathbf{1} \leq r^{t} \leq \Delta \mathbf{1}, t=1, \ldots, q$ (we leave this as an exercise). Since $\lambda \in C_{\lambda}$, by Caratheodory's Theorem 2.9, $\lambda=\sum_{t=1}^{q} \nu_{t} r^{t}$, and at most $m$ of the $\nu_{t}$ are positive, while the others are 0 . Let

$$
\lambda^{1}=\sum_{t=1}^{q}\left(\nu_{t}-\left\lfloor\nu_{t}\right\rfloor\right) r^{t}, \quad \lambda^{2}=\sum_{t=1}^{q}\left\lfloor\nu_{t}\right\rfloor r^{t} .
$$

Clearly $\lambda^{1}, \lambda^{2} \in C_{\lambda}$ and $\lambda=\lambda^{1}+\lambda^{2}$. Since $r^{1}, \ldots, r^{q}$ are integral vectors, $\lambda^{2}$ is integral. We show that $\lambda^{2} \neq 0$. Since at most $m$ of the $\nu_{t}$ are positive, and by definition $-\Delta \mathbf{1} \leq r^{t} \leq \Delta \mathbf{1}, t=1, \ldots, q$, then $-\Delta \mathbf{m} \leq \lambda^{1} \leq \Delta \mathbf{m}$. Thus $\lambda^{2} \neq 0$, as $\lambda$ violates (28). 
Theorem 6.5. (Cook, Kannan and Schrijver [17]) $P^{G M I}\left(=P^{S p l i t}\right)$ is a rational polyhedron.

Proof. By Lemma 5.1, for every $\lambda \in \mathbb{Q}^{m}, G M(\lambda)$ is a split inequality valid for $P^{\left(\pi^{\lambda}, \pi_{0}^{\lambda}\right)}$, where the split $\left(\pi^{\lambda}, \pi_{0}^{\lambda}\right) \in Z^{n+1}$ is defined by $(21)-(22)$. By Lemma 6.4, if $G M(\lambda)$ is irredundant for $P^{G M I}$ and not valid for $P$, then $\lambda$ satisfies (28). By Theorem $5.5, P^{G M I}=P^{S p l i t}$, thus any inequality valid for $P^{\left(\pi^{\lambda}, \pi_{0}^{\lambda}\right)}$ is valid for $P^{G M I}$. Therefore

$$
\begin{aligned}
& P^{G M I}=\bigcap_{\left(\pi^{\lambda}, \pi_{0}^{\lambda}\right) \in \mathbb{Z}^{n+1} \text { s.t. }} P^{\left(\pi^{\lambda}, \pi_{0}^{\lambda}\right)} . \\
& \lambda \text { satisfies (28) }
\end{aligned}
$$

Since the set $\left\{\lambda \in \mathbb{R}^{m}: \lambda\right.$ satisifes $\left.(28)\right\}$ is bounded, then the set $\left\{\left(\pi^{\lambda}, \pi_{0}^{\lambda}\right) \in\right.$ $\mathbb{Z}^{n+1}: \lambda$ satisifes (28)\} is finite. Therefore $P^{G M I}$ is the intersection of a finite number of polyhedra, hence it is a polyhedron.

A natural question is whether one can optimize a linear function over $P^{G M I}$ in polynomial time. It turns out that this problem is NP-hard (Caprara and Letchford [12], Cornuéjols and Li [19]). Equivalently, given a point $(\bar{x}, \bar{y}) \in P$, it is NP-hard to find a GMI inequality that cuts off $(\bar{x}, \bar{y})$ or show that none exists. A similar NP-hardness result was proved earlier by Eisenbrand [25] for the Chvátal closure $P^{C h}$.

Note that this is in contrast with the problem of finding a GMI inequality that cuts off a basic solution $(\bar{x}, \bar{y}) \in P \backslash S$. Indeed, any row of the simplex tableau where $\bar{x}_{j}$ is fractional generates a GMI inequality that cuts off $(\bar{x}, \bar{y})$.

Although it is NP-hard to optimize over the Chvátal closure, there are empirical results on its strength. For 24 instances from the MIPLIB library [8] (all the pure integer programs in MIPLIB 3 with nonzero integrality gap), Fischetti and Lodi [27] found that the Chvátal closure closes at least $63 \%$ of the integrality gap on average (The integrality gap is the difference between the values of the objective function when optimized over $\operatorname{conv}(S)$ and over $P$ respectively). Bonami, Cornuéjols, Dash, Fischetti, Lodi [10] found that the Chvátal closure closes at least $29 \%$ of the integrality gap on average on the remaining 41 MIPLIB instances (all the MIPLIB 3 instances that have at least one continuous variable and nonzero integrality gap).

The split closure and the GMI closure are identical. How tight is it in practice? Balas and Saxena [7] addressed this question by formulating the separation problem for the split closure as a parametric mixed integer linear program with a single parameter in the objective function and the right hand side. They found that the split closure closes $72 \%$ of the integrality 
gap on average on the MIPLIB instances. This experiment shows that the split closure is surprisingly strong. Finding deep split inequalities efficiently remains a challenging practical issue.

\section{$7 \quad$ Lift-and-Project}

In this section, we consider mixed 0,1 linear programs. These are mixed integer linear programs where the integer variables are only allowed to take the values 0 or 1 . It will be convenient to write mixed 0,1 linear programs in the form

$$
\begin{array}{rll}
\min & c x & \\
A x & \geq b & \\
x_{j} & \in\{0,1\} & \text { for } j=1, \ldots, n \\
x_{j} & \geq 0 & \text { for } j=n+1, \ldots, n+p,
\end{array}
$$

where the matrix $A \in \mathbb{Q}^{m \times(n+p)}$, the row vector $c \in \mathbb{Q}^{n+p}$ and the column vector $b \in \mathbb{Q}^{m}$ are data, and $x \in \mathbb{R}^{n+p}$ is a column vector of variables.

Consider the polyhedron $P:=\left\{x \in \mathbb{R}_{+}^{n+p}: A x \geq b\right\}$ and the mixed 0,1 linear set $S:=\left\{x \in\{0,1\}^{n} \times \mathbb{R}_{+}^{p}: A x \geq b\right\}$. Without loss of generality, throughout this section we assume that the constraints $A x \geq b$ include $-x_{j} \geq-1$ for $j=1, \ldots, n$, but not $x \geq 0$.

Balas, Ceria and Cornuéjols [4] study the following "lift-and-project" relaxation for $S$ : given an index $j \in\{1, \ldots, n\}$, let

$$
P_{j}=\operatorname{conv}\left\{\left(A x \geq b, x \geq 0, x_{j}=0\right) \cup\left(A x \geq b, x \geq 0, x_{j}=1\right)\right\} .
$$

Clearly $S \subseteq P_{j} \subseteq P$, so $P_{j}$ is a relaxation of $S$ tighter than $P$, and by definition it is the tightest possible among the relaxations that ignore the integrality of all the variables $x_{i}$ for $i \neq j$.

The set $\bigcap_{j=1}^{n} P_{j}$ is called the lift-and-project closure. It is a better approximation of $\operatorname{conv}(S)$ than $P$ :

$$
\operatorname{conv}(S) \subseteq \bigcap_{j=1}^{n} P_{j} \subseteq P .
$$

How much better is it in practice? Bonami and Minoux [11] performed computational experiments (see also Bonami's dissertation [9]). On 35 mixed 0,1 linear programs from MIPLIB, they found that the lift-and-project closure reduces the integrality gap by $37 \%$ on average. 


\subsection{Lift-and-project cuts}

Optimizing a linear function over $P_{j}$ amounts to solving a linear program. In fact, it is possible to express $P_{j}$ using Theorem 3.3 and then projecting onto the $x$-space. $P_{j}$ is the convex hull of the union of two polyhedra:

$$
\begin{aligned}
A x & \geq b & & A x & \geq b \\
x & \geq 0 & \text { and } & x & \geq 0 \\
-x_{j} & \geq 0 & & x_{j} & \geq 1
\end{aligned}
$$

By Theorem 3.3,

$$
P_{j}=\operatorname{proj}_{x}\left\{\begin{aligned}
A x^{0} & \geq b y_{0} \\
-x_{j}^{0} & \geq 0 \\
A x^{1} & \geq b y_{1} \\
x_{j}^{1} & \geq y_{1} \\
x^{0}+x^{1} & =x \\
y_{0}+y_{1} & =1 \\
x, x^{0}, x^{1}, y_{0}, y_{1} & \geq 0 .
\end{aligned}\right.
$$

Let $e_{j}$ denote the $j$-th unit vector. Using the projection theorem (Theorem 2.16), we get that $P_{j}$ is defined by the inequalities $\alpha x \geq \beta$ such that

$$
\begin{array}{rlrrrr}
\alpha & -u A & +u_{0} e_{j} & & \geq 0 \\
\alpha & & & -v A & -v_{0} e_{j} & \geq 0 \\
\beta-u b & & & \leq & \\
\beta & & & -v b & -v_{0} & \leq 0 \\
& & \leq & 0 \\
& u, & u_{0}, & v, & v_{0} & \geq 0 .
\end{array}
$$

The inequality $\alpha x \geq \beta$ is called a lift-and-project inequality. Clearly lift-and-project inequalities are special type of split inequalities, relative to splits of the type $x_{j} \leq 0$ or $x_{j} \geq 1$.

Given a fractional point $\bar{x}$, we can determine if there exists a lift-andproject inequality $\alpha x \geq \beta$ valid for $P_{j}$ that cuts off $\bar{x}$. In fact, this problem amounts to finding $\left(\alpha, \beta, u, u_{0}, v, v_{0}\right)$ satisfying (29) such that $\alpha \bar{x}-\beta<0$. In order to find a "best" cut in cone (29), one usually adds a normalization constaint to truncate the cone. We then obtain the following cut generating $L P$ : 


$$
\begin{array}{rrrrrrr}
\min \alpha \bar{x} & -\beta & & & & \\
\alpha & & -u A & +u_{0} e_{j} & & \\
\alpha & & & & -v A & -v_{0} e_{j} & \geq 0 \\
& \beta & -u b & & & & \leq 0 \\
& \beta & & -v b & -v_{0} & \leq 0 \\
& \sum_{i=1}^{m} u_{i} & +u_{0} & +\sum_{i=1}^{m} v_{i} & +v_{0} & =1 \\
& u, & u_{0}, & v, & v_{0} & \geq 0 .
\end{array}
$$

Balas and Perregaard [6] give a precise correspondence between the basic feasible solutions of (30) and the basic solutions (possibly infeasible) of the usual LP relaxation

(R) $\min \{c x: A x \geq b, x \geq 0\}$.

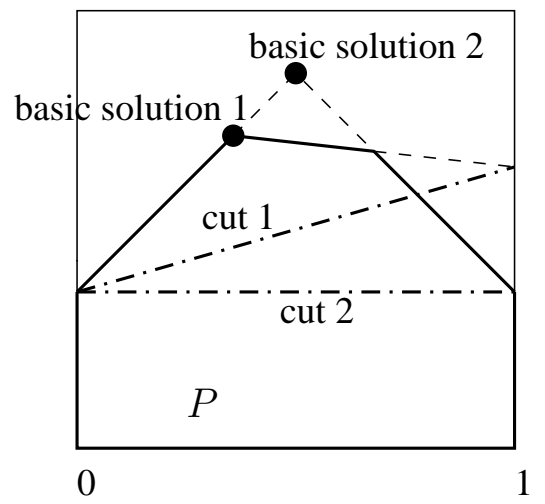

Figure 9: Correspondence between basic solutions and lift-and-project cuts

A geometric view of this correspondence may be helpful: The $n+p$ extreme rays emanating from a basic solution of $(\mathrm{R})$ intersect the hyperplanes $x_{j}=0$ and $x_{j}=1$ in $n+p$ points (some of these points may be at infinity). These points uniquely determine a hyperplane $\alpha x=\beta$ where $(\alpha, \beta)$ are associated with a basic feasible solution of the cut generating LP (30). For example, in Figure 9, cut 1 corresponds to the basic solution 1 of (R) and cut 2 corresponds to the basic (infeasible) solution 2 of (R).

Using the correspondence, Balas and Perregaard [6] show how simplex pivots in the cut generating LP (30) can be mimicked by pivots in (R). The major practical consequence is that the cut generating LP (30) need not be 
formulated and solved explicitly. A sequence of increasingly deep lift-andproject cuts can be computed by pivoting directly in $(\mathrm{R})$. We elaborate on these pivoting rules in section 7.3.

\subsection{Strengthened lift-and-project cuts}

Again we consider the mixed 0,1 linear set $S:=\left\{x \in\{0,1\}^{n} \times \mathbb{R}_{+}^{p}: A x \geq\right.$ $b\}$. We assume that the constraints $A x \geq b$ contain $-x_{j} \geq-1$ for $j=$ $1, \ldots, n$, but not $x \geq 0$. The cut generating LP (30) produces a lift-andproject inequality $\alpha x \geq \beta$ that is valid for $P_{j}$. The derivation only uses the integrality of variable $x_{j}$, not of the variables $x_{k}$ for $k=1, \ldots, n$ and $k \neq j$. Balas and Jeroslow [5] found a simple way to use the integrality of the other variables to strengthen the lift-and-project cut. This strengthening has the nice property that it is straightforward to implement once the cut generating LP (30) has been solved.

Note that, given $u, u_{0}, v, v_{0}$, the optimal values of $\alpha_{k}$ and $\beta$ in (30) are:

$$
\alpha_{k}= \begin{cases}\max \left(u a^{k}, v a^{k}\right) & \text { for } k \neq j \\ \max \left(u a^{j}-u_{0}, v a^{j}+v_{0}\right) & \text { for } k=j\end{cases}
$$

where $a^{k}$ denotes the $k$-th column of $A$, and

$$
\beta=\min \left(u b, v b+v_{0}\right) .
$$

To strengthen the inequality $\alpha x \geq \beta$, one can try to decrease the coefficients $\alpha_{k}$. Balas and Jeroslow [5] found a way to do just that by using the integrality of the variables $x_{k}$ for $k=1, \ldots, n$.

Theorem 7.1. (Balas and Jeroslow [5]) Let $\bar{x}$ satisfy $A x \geq b, x \geq 0$. Given an optimal solution $u, u_{0}, v, v_{0}$ of the cut generating $L P(30)$, define $m_{k}=$ $\frac{v a^{k}-u a^{k}}{u_{0}+v_{0}}$

$$
\alpha_{k}= \begin{cases}\min \left(u a^{k}+u_{0}\left\lceil m_{k}\right\rceil, v a^{k}-v_{0}\left\lfloor m_{k}\right\rfloor\right) & \text { for } k=1, \ldots, n \\ \max \left(u a^{k}, v a^{k}\right) & \text { for } k=n+1, \ldots, n+p\end{cases}
$$

and $\beta=\min \left(u b, v b+v_{0}\right)$. Then the inequality $\alpha x \geq \beta$ is valid for $\operatorname{conv}(S)$.

Proof. For $\pi \in \mathbb{Z}^{n}$, the following disjunction is valid for $\operatorname{conv}(S)$ :

$$
\text { either } \sum_{k=1}^{n} \pi_{k} x_{k} \geq 0 \quad \text { or } \quad-\sum_{k=1}^{n} \pi_{k} x_{k} \geq 1 \text {. }
$$


Let us repeat the derivation of (30) with this disjunction in place of $-x_{j} \geq 0$ or $x_{j} \geq 1$ as before. We consider the union of

$$
\begin{aligned}
& A x \geq b \quad A x \geq b \\
& x \geq 0 \text { and } \quad x \geq 0 \\
& \sum_{k=1}^{n} \pi_{k} x_{k} \geq 0 \quad-\sum_{k=1}^{n} \pi_{k} x_{k} \geq 1 .
\end{aligned}
$$

Using Theorem 3.3 and the projection theorem (Theorem 2.16), we get that any inequality $\alpha x \geq \beta$ that satisfies

$$
\begin{array}{rrrrr}
\alpha & -u A & -u_{0}\left(\sum_{k=1}^{n} \pi_{k} e_{k}\right) & & \\
& & & \\
\alpha & & & \\
\beta-u b & & +v_{0}\left(\sum_{k=1}^{n} \pi_{k} e_{k}\right) & \geq 0 \\
\beta & & & \leq 0 \\
& & -v b & -v_{0} & \leq 0 \\
& u_{0}, & v, & v_{0} & \geq 0
\end{array}
$$

is valid for $\operatorname{conv}(S)$. We can choose $u, u_{0}, v, v_{0}$ to be an optimal solution of the original cut generating LP (30). This implies that, for $k=1, \ldots, n$, we can choose $\alpha_{k}=\max \left(u a^{k}+u_{0} \pi_{k}, v a^{k}-v_{0} \pi_{k}\right)$. Smaller coefficients $\alpha_{k}$ produce stronger inequalities since the variables are nonnegative. What is the best choice of $\pi_{k} \in \mathbb{Z}$ to get a small $\alpha_{k}$ ? It is obtained by equating $u a^{k}+u_{0} \pi_{k}$ and $v a^{k}-v_{0} \pi_{k}$, which yields the value $m_{k}$ in the statement of the theorem (both $u_{0}$ and $v_{0}$ are strictly positive since otherwise $\alpha x \geq \beta$ is valid for $P$, contradicting that it is a cut for $\bar{x}$ ), and then rounding this value $m_{k}$ either up or down since $\pi_{k}$ must be integer. The best choice is the minimum stated in the theorem.

Bonami and Minoux [11] found that applying the Balas-Jeroslow strengthening step improves the average gap closed by an additional $8 \%$, as compared to the lift-and-project closure, on the 35 MIPLIB instances in their experiment. Specifically, the integrality gap closed goes from $37 \%$ to $45 \%$. The time to perform the strengthening step is negligible.

\subsection{Improving mixed integer Gomory cuts by lift-and-project}

In this section we discuss the correspondence between basic feasible solutions of the cut generating LP (30) and basic solutions (possibly infeasible) of the usual LP relaxation ( $\mathrm{R}$ ) introduced in Section 7.1. The simplex tableaux of (30) and (R) will be referred to as large and small respectively.

Let

$$
x_{j}=a_{j 0}-\sum_{h \in J} a_{j h} x_{h}
$$


be a row of the small optimal simplex tableau such that $0<a_{j 0}<1$. The GMI cut from this row is equivalent to the strengthened lift-and-project cut from some basic feasible solution of (30), where index $j$ in (30) is the same as in (32). To identify this solution, partition $J$ into subsets $M_{1}$ and $M_{2}$, such that $h \in M_{1}$ if $a_{j h}<0$, and $h \in M_{2}$ if $a_{j h}>0(h \in J$ such that $a_{j h}=0$ can go into either subset). Then eliminating $\alpha, \beta$ from (30), the $n$ columns indexed by $M_{1} \cup M_{2}$ together with the two columns indexed by $u_{0}$ and $v_{0}$ define a feasible basis of the resulting system of $n+2$ equations. The strengthened lift-and-project cut associated with this basic feasible solution to (30) is equivalent to the GMI cut from (32).

To evaluate the GMI cut generated from the small simplex tableau (32) as a lift-and-project cut, we calculate the reduced costs in the large tableau of the nonbasic variables of the above solution to (30). Each row $x_{i}$ of the small tableau corresponds to a pair of columns of the large tableau, associated with variables $u_{i}$ and $v_{i}$. The reduced costs $r\left(u_{i}\right), r\left(v_{i}\right)$ of these variables in the large tableau are known simple functions of the entries $a_{i h}$ and $a_{j h}$, for $h \in J$, of rows $j$ and $i$ of the small tableau. If they are all nonnegative, the current large tableau is optimal, hence the GMI cut from (32) cannot be improved. Otherwise, the cut can be improved by executing a pivot in a row $i$ of the small tableau, such that $r\left(u_{i}\right)<0$ or $r\left(v_{i}\right)<0$.

To identify the nonbasic variable $x_{k}$ to replace $x_{i}$ in the basis of the small tableau, we calculate for each $h \in J$ the objective function value $f\left(a_{i h}\right)$ of (30) resulting from the corresponding exchange in the large tableau. This value is a known simple function of the ratio $a_{j h} / a_{i h}$ and of the coefficients of rows $j$ and $i$ of the small tableau. Any column $h$ for which $f\left(a_{i h}\right)<0$ is a candidate for an improving pivot, and the most negative value indicates the best column $k$.

Executing the pivot in the small tableau that exchanges $x_{i}$ for $x_{k}$ yields a new simplex tableau (whose solution is typically infeasible), whose $j$-th row (the same $j$ as before!) is of the form

$$
x_{j}=a_{j 0}+t a_{i 0}-\sum_{h \in J \cup i \backslash k}\left(a_{j h}+t a_{i h}\right) x_{h},
$$

with $t:=a_{j k} / a_{i k}$. The GMI cut from (33) is then stronger than the one from (32), in the sense that it cuts off the LP optimum of (R) by a larger amount.

These steps can then be repeated with (33) replacing (32) for as long as improvements are possible.

Practical experience shows that in about three quarters of the cases GMI 
cuts from the optimal simplex tableau can be improved by the pivoting procedure described above. On the other hand, improvements beyond 10 pivots are not frequent, and beyond 20 pivots they are very rare.

This procedure was extensively tested and has been incorporated into the mixed integer module of XPRESS, with computational results reported in $[42]$.

\subsection{Sequential convexification}

Theorem 7.2. (Balas [2]) $P_{n}\left(P_{n-1}\left(\ldots P_{2}\left(P_{1}\right) \ldots\right)\right)=\operatorname{conv}(S)$.

Before proving Theorem 7.2, we need a lemma. Let $H \subseteq \mathbb{R}^{n}$ be a hyperplane and $S \subseteq \mathbb{R}^{n}$. In general, $\operatorname{conv}(S) \cap H \neq \operatorname{conv}(S \cap H)$, as shown by the example where $S$ consists of two points not in $H$ but the line segment connecting them intersects $H$. The following lemma shows that equality holds when $S$ lies entirely in one of the closed half spaces defined by the hyperplane $H$ (see Figure 10).

Lemma 7.3. Let $H:=\left\{x \in \mathbb{R}^{n}: a x=b\right\}$ be a hyperplane and $S \subseteq\{x$ : $a x \leq b\}$. Then $\operatorname{conv}(S) \cap H=\operatorname{conv}(S \cap H)$.

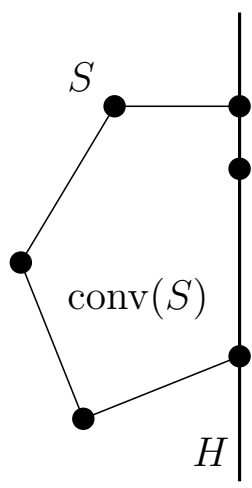

Figure 10: Illustration of Lemma 7.3

Proof. Clearly $\operatorname{conv}(S \cap H) \subseteq \operatorname{conv}(S)$ and $\operatorname{conv}(S \cap H) \subseteq H$ so $\operatorname{conv}(S \cap$ $H) \subseteq \operatorname{conv}(S) \cap H$.

We show $\operatorname{conv}(S) \cap H \subseteq \operatorname{conv}(S \cap H)$. Let $x \in \operatorname{conv}(S) \cap H$. This means $a x=b$ and $x=\sum_{i=1}^{k} \lambda_{i} x^{i}$ where $x^{1}, \ldots, x^{k} \in S, \lambda \geq 0$ and $\sum_{i=1}^{k} \lambda_{i}=1$.

$$
b=a x=\sum_{i=1}^{k} \lambda_{i} a x^{i} \leq \sum_{i=1}^{k} \lambda_{i} b=b
$$


where the inequality follows from $a x^{i} \leq b$ and $\lambda_{i} \geq 0$. Relation (34) implies that these inequalities are in fact equations, i.e. $a x^{i}=b$ for $i=1, \ldots, k$. Therefore $x^{i} \in S \cap H$. This implies $x \in \operatorname{conv}(S \cap H)$.

Proof of Theorem 7.2. By induction. Let $S_{t}:=\left\{x \in\{0,1\}^{t} \times \mathbb{R}_{+}^{n-t+p}: A x \geq\right.$ $b\}$. We want to show $P_{t}\left(P_{t-1}\left(\ldots P_{2}\left(P_{1}\right) \ldots\right)\right)=\operatorname{conv}\left(S_{t}\right)$. By definition, this is true for $t=1$, so consider $t \geq 2$. Suppose that this is true for $t-1$. By the induction hypothesis we have

$$
\begin{gathered}
P_{t}\left(P_{t-1}\left(\ldots P_{2}\left(P_{1}\right) \ldots\right)\right)=P_{t}\left(\operatorname{conv}\left(S_{t-1}\right)\right) \\
=\operatorname{conv}\left(\operatorname{conv}\left(S_{t-1}\right) \cap\left\{x_{t}=0\right\}\right) \cup\left(\operatorname{conv}\left(S_{t-1}\right) \cap\left\{x_{t}=1\right\}\right) .
\end{gathered}
$$

By Lemma 7.3, $\operatorname{conv}\left(S_{t-1}\right) \cap\left\{x_{t}=0\right\}=\operatorname{conv}\left(S_{t-1} \cap\left\{x_{t}=0\right\}\right)$ and $\operatorname{conv}\left(S_{t-1}\right) \cap\left\{x_{t}=1\right\}=\operatorname{conv}\left(S_{t-1} \cap\left\{x_{t}=1\right\}\right)$. Thus

$$
\begin{aligned}
P_{t}\left(P_{t-1}\left(\ldots P_{2}\left(P_{1}\right) \ldots\right)\right) & =\operatorname{conv}\left(\left(S_{t-1} \cap\left\{x_{t}=0\right\}\right) \cup\left(S_{t-1} \cap\left\{x_{t}=1\right\}\right)\right) \\
& =\operatorname{conv}\left(S_{t}\right) .
\end{aligned}
$$

\section{Rank}

\subsection{Chvátal Rank}

In this section, we consider a pure integer set $S:=P \cap \mathbb{Z}^{n}$ where $P:=\{x \in$ $\left.\mathbb{R}^{n}: A x \leq b\right\}$ is a rational polyhedron. We denote $\operatorname{conv}(S)$ by $P_{I}$. The Chvátal closure $P^{C h}$ introduced in Section 4.1 will be denoted by $P^{(1)}$ in this section. We can iterate the closure process to obtain the Chvátal closure of $P^{(1)}$. We denote by $P^{(2)}$ this second Chvátal closure. Iteratively, we define the $t$ th Chvátal closure of $P$ to be the Chvátal closure of $P^{(t-1)}$, for $t \geq 2$ integer. An inequality that is valid for $P^{(t)}$ but not $P^{(t-1)}$ is said to have Chvátal rank $t$. Are there inequalities of arbitrary large Chvátal rank or is there a value $t$ after which $P^{(t)}=P^{(t+1)}$ ? The main result of this section is that the second statement is the correct one. In fact, we will prove that there exists a finite $t$ such that $P^{(t)}=P_{I}$. Therefore, every valid inequality for $P_{I}:=\operatorname{conv}(S)$ has a bounded Chvátal rank. This result for the pure integer case is in contrast with the situation for the mixed case, as we will see in the next section.

We will need the following theorem, whose proof can be found in textbooks such as [45]. 
Theorem 8.1. (Integer Farkas Lemma or Kronecker Approximation Theorem) Let $A$ be a rational matrix and $b$ a rational vector. The system $A x=b$ has an integral solution if and only if for every rational vector $u$ such that $u A$ is integral, $u b$ is an integer.

Given a set $P \subset \mathbb{R}^{n}$, we denote by aff $(P)$ the affine hull of $P$, that is the minimal affine subspace of $\mathbb{R}^{n}$ containing $P$.

Lemma 8.2. Let $P \subseteq \mathbb{R}^{n}$ be a nonempty rational polyhedron such that $\operatorname{aff}(P) \cap \mathbb{Z}^{n} \neq \emptyset$. If $P_{I}=\emptyset$, then $\operatorname{dim}(\operatorname{rec}(P))<\operatorname{dim}(P)$.

Proof. Let $d=\operatorname{dim}(P)=\operatorname{dim}(\operatorname{aff}(P))$. Suppose, by contradiction, that $P_{I}=\emptyset$ and there are $d$ linearly independent integral vectors $r^{1}, \ldots, r^{d} \in$ $\operatorname{rec}(P)$. Let $z \in P$. Since $\operatorname{aff}(P) \cap \mathbb{Z}^{n} \neq \emptyset$, and $z+r^{1}, \ldots, z+r^{d}$ is a basis of aff $(P)$, there exist $\mu_{1}, \ldots, \mu_{d}$ such that $z+\sum_{i=1}^{d} \mu_{i} r^{i} \in \mathbb{Z}^{n}$. Thus $z+\sum_{i=1}^{d}\left(\mu_{i}-\left\lfloor\mu_{i}\right\rfloor\right) r^{i}$ is an integral point in $P$, contradicting the fact that $P_{I}=\emptyset$.

A consequence of the above lemma is that every rational polyhedron having full-dimensional recession cone contains an integer point.

Lemma 8.3. Let $P \subseteq \mathbb{R}^{n}$ be a rational polyhedron such that aff $(P) \cap \mathbb{Z}^{n} \neq \emptyset$. Then $P_{I}=\{x: A x \leq b\} \cap \operatorname{aff}(P)$ for some integral $A$ and $b$ such that, for every row $a_{i}$ of $A$,

1. $a_{i}$ is not orthogonal to aff $(P)$;

2. there exists $d_{i} \in \mathbb{R}$ such that $a_{i} x \leq d_{i}$ is valid for $P$.

Proof. Assume first $P_{I} \neq \emptyset$. Then clearly there exist an integral matrix $A$ and an integral vector $b$ such that $P_{I}=\{x: A x \leq b\} \cap \operatorname{aff}(P)$ and no row of $A$ is orthogonal to $\operatorname{aff}(P)$. We prove 2$)$ : Since $\operatorname{rec}\left(P_{I}\right)=\operatorname{rec}(P)$ by Theorem 2.19, for every row $a_{i}, d_{i}=\max \left\{a_{i} x: x \in P\right\}$ is finite, thus $a_{i} x \leq d_{i}$ is valid for $P$.

Assume now $P_{I}=\emptyset$. By standard linear algebra, $\operatorname{aff}(P)=z+L$ where $z \in P$ and $L$ is a linear subspace of $\mathbb{R}^{n}$ such that $\operatorname{dim}(L)=\operatorname{dim}(P)$. Notice that $\operatorname{rec}(P) \subset L$. By Lemma 8.2, $\operatorname{dim}(\operatorname{rec}(P))<\operatorname{dim}(P)$, thus there exists an integral $a \in L$ such that $a$ is orthogonal to $\operatorname{rec}(P)$. Thus both $u=$ $\max \{a x: x \in P\}$ and $l=\min \{a x: x \in P\}$ are finite, hence $P_{I}=\{x:$ $a x \leq-1,-a x \leq 0\}=\emptyset, a,-a$ are not orthogonal to aff $(P)$, and $a x \leq u$, $-a x \leq-l$ are valid for $P$.

Lemma 8.4. Let $P$ be a rational polyhedron and $F$ a nonempty face of $P$. Then $F^{(s)}=P^{(s)} \cap F$ for every $s \in \mathbb{Z}_{+}$. 
Proof. It suffices to show that $F^{(1)}=P^{(1)} \cap F$. This is a consequence of the following statement, which we prove next:

If cx $\leq\lfloor d\rfloor$ is a Chvátal inequality for $F$, there is a Chvátal inequality $c^{*} x \leq\left\lfloor d^{*}\right\rfloor$ for $P$ such that $F \cap\{x: c x \leq\lfloor d\rfloor\}=F \cap\left\{x: c^{*} x \leq\left\lfloor d^{*}\right\rfloor\right\}$.

Since $P$ is rational, by Theorem 2.22 , we can write $P$ as $\left\{x: A^{\prime} x \leq\right.$ $\left.b^{\prime}, A^{\prime \prime} x \leq b^{\prime \prime}\right\}$, where $A^{\prime}, A^{\prime \prime}, b^{\prime}, b^{\prime \prime}$ are integral, so that $F=\left\{x: A^{\prime} x \leq\right.$ $\left.b^{\prime}, A^{\prime \prime} x=b^{\prime \prime}\right\}$. We can assume that $d=\max \{c x: x \in F\}$. By the duality theorem 2.6 there exist vectors $y^{\prime}, y^{\prime \prime}$ such that

$$
y^{\prime} A^{\prime}+y^{\prime \prime} A^{\prime \prime}=c, y^{\prime} b^{\prime}+y^{\prime \prime} b^{\prime \prime}=d, y^{\prime} \geq 0
$$

Note that $y^{\prime \prime}$ is unrestricted in sign. To obtain a Chvátal inequality for $P$, we have to use nonnegative multipliers. Define $c^{*}$ and $d^{*}$ as:

$$
c^{*}=y^{\prime} A^{\prime}+\left(y^{\prime \prime}-\left\lfloor y^{\prime \prime}\right\rfloor\right) A^{\prime \prime}, d^{*}=y^{\prime} b^{\prime}+\left(y^{\prime \prime}-\left\lfloor y^{\prime \prime}\right\rfloor\right) b^{\prime \prime} .
$$

The multipliers $y^{\prime}$ and $y^{\prime \prime}-\left\lfloor y^{\prime \prime}\right\rfloor$ are nonnegative. We have $c^{*}=c-$ $\left.\left.\left(\left\lfloor y^{\prime \prime}\right\rfloor\right) A^{\prime \prime}\right), d^{*}=d-\left(\left\lfloor y^{\prime \prime}\right\rfloor\right) b^{\prime \prime}\right)$. Since $A^{\prime \prime}$ is an integral matrix and $b^{\prime \prime}, c$ are integral vectors, then $c^{*}$ is integral and $\lfloor d\rfloor=\left\lfloor d^{*}\right\rfloor-\left(\left\lfloor y^{\prime \prime}\right\rfloor\right) b^{\prime \prime}$. So $c^{*} x \leq\left\lfloor d^{*}\right\rfloor$ is a Chvátal inequality for $P$ and $F \cap\left\{x: c^{*} x \leq\left\lfloor d^{*}\right\rfloor\right\}=F \cap\left\{x:\left\lfloor y^{\prime \prime}\right\rfloor A^{\prime \prime} x=\right.$ $\left.\left\lfloor y^{\prime \prime}\right\rfloor b^{\prime \prime}, c^{*} x \leq\left\lfloor d^{*}\right\rfloor\right\} F \cap\{x: c x \leq\lfloor d\rfloor\}$.

Theorem 8.5. (Chvátal [13], Schrijver [44]) Let $P$ be a rational polyhedron. Then there exists $t \in \mathbb{Z}_{+}$such that $P^{(t)}=P_{I}$.

Proof. The proof is by induction on $d=\operatorname{dim}(P)$, the cases $d=-1, d=0$ being trivial. If aff $(P) \cap \mathbb{Z}^{n}=\emptyset$, by Theorem 8.1 there exists an integral vector $a$ and a scalar $d \notin \mathbb{Z}$ such that $P \subseteq\{x: a x=d\}$, hence $P_{I}=\emptyset=\{x$ : $a x \leq\lfloor d\rfloor,-a x \leq-\lceil d\rceil\}=P^{(1)}$. Therefore we may assume aff $(P) \cap \mathbb{Z}^{n} \neq \emptyset$. By Lemma 8.3, $P_{I}=\{x: A x \leq b\} \cap \operatorname{aff}(P)$ for some integral $A$ and $b$ such that, for every row $a_{i}$ of $A, a_{i}$ is not orthogonal to aff $(P)$ and $a_{i} x \leq d_{i}$ is valid for $P$ for some $d_{i} \in \mathbb{R}$.

We only need to show that, for any row $a_{i}$ of $A$, there exists a nonnegative integer $t$ such that the inequality $a_{i} x \leq b_{i}$ is valid for $P^{(t)}$. Suppose not, then, since $a_{i} x \leq d_{i}$ is valid for $P$, there exists an integer $d>b_{i}$ and $r \in \mathbb{Z}_{+}$ such that, for every $s \geq r, a_{i} x \leq d$ is valid for $P^{(s)}$ but $a_{i} x \leq d-1$ is not valid for $P^{(s)}$. Then $F=P^{(r)} \cap\left\{x: a_{i} x=d\right\}$ is a face of $P^{(r)}$ and $F_{I}=\emptyset$. Since $a_{i}$ is not orthogonal to $\operatorname{aff}(P), \operatorname{dim}(F)<\operatorname{dim}(P)$, therefore, by induction, there exists $h$ such that $F^{(h)}=\emptyset$. By Lemma 8.4, $F^{(h)}=P^{(r+h)} \cap F$, hence $a_{i} x<d$ for every $x \in P^{(r+h)}$, therefore $a_{i} x \leq \beta-1$ is valid for $P^{(r+h+1)}$, contradicting the choice of $d$ and $r$. 


\subsection{Split Rank}

Let $P:=\left\{(x, y) \in \mathbb{R}^{n} \times \mathbb{R}^{p}: A x+G y \leq b\right\}$ and let $S:=P \cap\left(\mathbb{Z}^{n} \times \mathbb{R}^{p}\right)$. In this section, we denote the split closure $P^{\text {Split }}$ of $P$ by $P^{1}$.

For $k \geq 2, P^{k}$ denotes the split closure relative to $P^{k-1}$ and it is called the $k$-th split closure relative to $P$. It follows from Theorem 6.5 that $P^{k}$ is a polyhedron. Unlike for the pure integer case, there is in general no finite $r$ such that $P^{r}=\operatorname{conv}(S)$ in the mixed integer case, as shown by the following example [17].

Example 8.6. Let $S:=\left\{(x, y) \in \mathbb{Z}_{+}^{2} \times \mathbb{R}_{+}: x_{1} \geq y, x_{2} \geq y, x_{1}+x_{2}+2 y \leq\right.$ $2\}$. Starting from $P:=\left\{\left(x_{1}, x_{2}, y\right) \in \mathbb{R}_{+}^{3}: x_{1} \geq y, x_{2} \geq y, x_{1}+x_{2}+2 y \leq\right.$ $2\}$, we claim that there is no finite $r$ such that $P^{r}=\operatorname{conv}(S)$.

To see this, note that $P$ is a simplex with vertices $O=(0,0,0), A=$ $(2,0,0), B=(0,2,0)$ and $C=\left(\frac{1}{2}, \frac{1}{2}, \frac{1}{2}\right)$ (see Figure11). $S$ is contained in the plane $y=0$. More generally, consider a simplex $P$ with vertices $O, A, B$ and $C=\left(\frac{1}{2}, \frac{1}{2}, t\right)$ with $t>0$. Let $C_{1}=C$, let $C_{2}$ be the point on the edge from $C$ to $A$ with coordinate $x_{1}=1$ and $C_{3}$ the point on the edge from $C$ to $B$ with coordinate $x_{2}=1$. Observe that no split disjunction removes all three points $C_{1}, C_{2}, C_{3}$. Let $Q_{i}$ be the intersection of all split inequalities that do not cut off $C_{i}$. All split inequalities belong to at least one of these three sets, thus $P^{1}=Q_{1} \cap Q_{2} \cap Q_{3}$. Let $S_{i}$ be the simplex with vertices $O, A, B, C_{i}$. Clearly, $S_{i} \subseteq Q_{i}$. Thus $S_{1} \cap S_{2} \cap S_{3} \subseteq P^{1}$. It is easy to verify that $\left(\frac{1}{2}, \frac{1}{2}, \frac{t}{3}\right) \in S_{i}$ for $i=1,2$ and 3. Thus $\left(\frac{1}{2}, \frac{1}{2}, \frac{t}{3}\right) \in P^{1}$. By induction, $\left(\frac{1}{2}, \frac{1}{2}, \frac{t}{3^{k}}\right) \in P^{k}$.

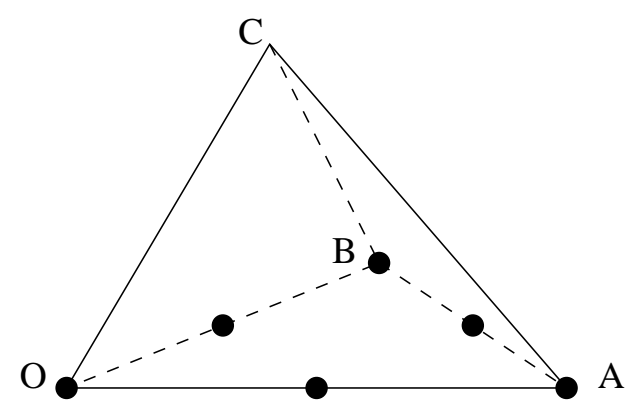

Figure 11: Example showing that the split rank can be unbounded

Remark 8.7. For mixed 0,1 programs, Theorem 7.2 implies that $P^{n}=$ $\operatorname{conv}(S)$. 
Example 8.8. Cornuéjols and Li [18] observed that the $n$-th split closure is needed for 0,1 programs, i.e. there are examples where $P^{k} \neq \operatorname{conv}(S)$ for all $k<n$. They use the following well-known polytope studied by Chvátal, Cook, and Hartmann [14]:

$$
P_{C C H}:=\left\{x \in[0,1]^{n}: \sum_{j \in J} x_{j}+\sum_{j \notin J}\left(1-x_{j}\right) \geq \frac{1}{2}, \text { for all } J \subseteq\{1,2, \cdots, n\}\right\}
$$

Let $F_{j}$ be the set of all vectors $x \in \mathbb{R}^{n}$ such that $j$ components of $x$ are $\frac{1}{2}$ and each of the remaining $n-j$ components are equal to 0 or 1 . The polytope $P_{C C H}$ is the convex hull of $F_{1}$.

Lemma 8.9. If a polyhedron $P \subseteq \mathbb{R}^{n}$ contains $F_{j}$, then its split closure $P^{1}$ contains $F_{j+1}$.

Proof. It suffices to show that, for every $\left(\pi, \pi_{0}\right) \in \mathbb{Z}^{n} \times \mathbb{Z}$, the polyhedron $\Pi=\operatorname{conv}\left(\left(P \cap\left\{x: \pi x \leq \pi_{0}\right\}\right) \cup\left(P \cap\left\{x: \pi x \geq \pi_{0}+1\right\}\right)\right)$ contains $F_{j+1}$. Let $v \in F_{j+1}$ and assume w.l.o.g. that the first $j+1$ elements of $v$ are equal to $\frac{1}{2}$. If $\pi v \in \mathbb{Z}$, then clearly $v \in \Pi$. If $\pi v \notin \mathbb{Z}$, then at least one of the first $j+1$ components of $\pi$ is nonzero. Assume w.l.o.g. that $\pi_{1}>0$. Let $v_{1}, v_{2} \in F_{j}$ be equal to $v$ except for the first component which is 0 and 1 respectively. Notice that $v=\frac{v_{1}+v_{2}}{2}$. Clearly, each of the intervals $\left[\pi v_{1}, \pi v\right]$ and $\left[\pi v, \pi v_{2}\right]$ contains an integer. Since $\pi x$ is a continuous function, there are points $\tilde{v}_{1}$ on the line segment $\operatorname{conv}\left(v, v_{1}\right)$ and $\tilde{v}_{2}$ on the line segment $\operatorname{conv}\left(v, v_{2}\right)$ with $\pi \tilde{v}_{1} \in \mathbb{Z}$ and $\pi \tilde{v}_{2} \in \mathbb{Z}$. This means that $\tilde{v}_{1}$ and $\tilde{v}_{2}$ are in $\Pi$. Since $v \in \operatorname{conv}\left(\tilde{v}_{1}, \tilde{v}_{2}\right)$, this implies $v \in \Pi$.

Starting from $P=P_{C C H}$ and applying the lemma recursively, it follows that the $(n-1)$-st split closure relative to $P_{C C H}$ contains $F_{n}$, which is nonempty. Since $\operatorname{conv}\left(P_{C C H} \cap\{0,1\}^{n}\right)$ is empty, the $n$-th split closure is needed to obtain conv $\left(P_{C C H} \cap\{0,1\}^{n}\right)$. End of Example 8.8.

Remark 8.10. In view of Example 8.6 showing that no bound may exist on the split rank when the integer variables are general, and Remark 8.7 showing that the rank is always bounded when they are 0,1 valued, one is tempted to convert general integer variables into 0,1 variables. For a bounded integer variable $0 \leq x \leq u$, there are several natural transformations:

(i) a binary expansion of $x$ (see Owen and Mehrotra [41]);

(ii) $x=\sum_{i=1}^{u} i z_{i}, \sum z_{i} \leq 1, z_{i} \in\{0,1\}$ (see Sherali and Adams [46] and Köppe, Louveaux and Weismantel [35]);

(iii) $x=\sum_{i=1}^{u} z_{i}, z_{i} \leq z_{i-1}, z_{i} \in\{0,1\}$ (see Roy [43]).

More studies are needed to determine whether any practical benefit can be gained from such transformations. 


\section{References}

[1] K. Andersen, G. Cornuéjols and Y. Li, Split closure and intersection cuts, Mathematical Programming A 102 (2005) 457-493.

[2] E. Balas, Disjunctive programming: properties of the convex hull of feasible points, GSIA Management Science Research Report MSRR 348, Carnegie Mellon University (1974), published as invited paper in Discrete Applied Mathematics 89 (1998) 1-44.

[3] E. Balas, Disjunctive programming and a hierarchy of relaxations for discrete optimization problems, SIAM Journal on Algebraic and Discrete Methods 6 (1985) 466-486.

[4] E. Balas, S. Ceria and G. Cornuéjols, A lift-and-project cutting plane algorithm for mixed 0-1 programs, Mathematical Programming 58 (1993) $295-324$.

[5] E. Balas and R. Jeroslow, Strengthening cuts for mixed integer programs, European Journal of Operations Research 4 (1980) 224-234.

[6] E. Balas and M. Perregaard, A Precise correspondence between lift-andproject cuts, simple disjunctive cuts and mixed integer Gomory cuts for 0-1 programming, Mathematical Programming B 94 (2003) 221-245.

[7] E. Balas and A. Saxena, Optimizing over the split closure, Mathematical Programming A 113 (2008) 219-240.

[8] R.E. Bixby, S. Ceria, C.M. McZeal and M.W.P. Savelsbergh, An updated mixed integer programming library: MIPLIB 3.0, Optima 58 (1998) 12-15.

[9] P. Bonami, Etude et mise en oeuvre d'approches polyédriques pour la résolution de programmes en nombres entiers ou mixtes généraux, $\mathrm{PhD}$ Thesis, Université de Paris 6 (2003).

[10] P. Bonami, G. Cornuéjols, S. Dash, M. Fischetti and A. Lodi, Projected Chvátal-Gomory cuts for mixed integer linear programs, Mathematical Programming A 113 (2008) 241-257.

[11] P. Bonami and M. Minoux, Using rank-1 lift-and-project closures to generate cuts for 0-1 MIPs, a computational investigation, Discrete Optimization 2 (2005) 288-307. 
[12] A. Caprara and A.N. Letchford, On the separation of split cuts and related inequalities, Mathematical Programming B 94 (2003) 279-294.

[13] V. Chvátal, Edmonds polytopes and a hierarchy of combinatorial optimization, Discrete Mathematics 4 (1973) 305-337.

[14] V. Chvátal, W. Cook, and M. Hartmann, On cutting-plane proofs in combinatorial optimization, Linear Algebra and its Applications $114 / 115$ (1989) 455-499.

[15] M. Conforti, M. Di Summa and G. Zambelli, Minimally Infeasible Set Partitioning Problems with Balanced Constraints, Mathematics of Operations Research 32 (2007) 497-507.

[16] S.A. Cook, The complexity of theorem-proving procedures, in: Proceedings of the Third Annual ACM Symposium on the Theory of Computing (Shaker Heights, Ohio 1971), ACM, New York (1971) 151-158.

[17] W. Cook, R. Kannan and A. Schrijver, Chvátal closures for mixed integer programming problems, Mathematical Programming 47 (1990) $155-174$.

[18] G. Cornuéjols and Y. Li, On the rank of mixed 0,1 polyhedra, Mathematical Programming A 91 (2002) 391-397.

[19] G. Cornuéjols and Y. Li, A connection between cutting plane theory and the geometry of numbers, Mathematical Programming A 93 (2002) $123-127$.

[20] G.B. Dantzig, Maximization of a linear function of variables subject to linear inequalities, in Activity Analysis of Production and allocation, T.C. Koopmans ed., Wiley N.Y. (1951) 339-347.

[21] G. Dantzig. R. Fulkerson and S. Johnson, Solution of a large-scale traveling-salesman problem, Operations Research 2 (1954) 393-410.

[22] S. Dash, O. Günlük and A. Lodi, MIR closures of polyhedral sets, IBM Research Tech. Report (2007).

[23] J. Edmonds, Paths, trees, and flowers, Canadian Journal of Mathematics 17 (1965) 449-467.

[24] J. Edmonds, Systems of distinct representatives and linear algebra, Journal of Research of the National Bureau of Standards B 71 (1967) 241-245. 
[25] F. Eisenbrand, On the membership problem for the elementary closure of a polyhedron, Combinatorica 19 (1999) 297-300.

[26] Gy. Farkas, On the applications of the mechanical principle of Fourier, Mathematikai és Természettudományi Értesotö 12 (1894) 457-472.

[27] M. Fischetti and A. Lodi, Optimizing over the first Chvátal closure, Mathematical Programming B 110 (2007) 3-20.

[28] J.B.J. Fourier, Solution d'une question particulière du calcul des inégalités, Nouveau Bulletin des Sciences par la Société Philomatique de Paris (1826) 317-319.

[29] C.F. Gauss, Theoria Motus Corporum Coelestium in Sectionibus Conicis Solem Ambientium, F. Perthes \& J.H. Besser, Hamburg (1809).

[30] M.X. Goemans and D.P. Williamson, Improved approximation algorithms for maximum cut and satisfiability problems using semidefinite programming, Journal of the ACM 42 (1995) 1115-1145.

[31] R.E. Gomory, Outline of an algorithm for integer solutions to linear programs, Bulletin of the American Mathematical Society 64 (1958) $275-278$.

[32] R.E. Gomory, An algorithm for integer solutions to linear programs, Recent Advances in Mathematical Programming, R.L. Graves and P. Wolfe eds., McGraw-Hill, New York (1963) 269-302.

[33] N. Karmarkar, A new polynomial-time algorithm for linear programming, Combinatorica 4 (1984) 373-395.

[34] L.G. Khachiyan, A polynomial algorithm in linear programming, Soviet Mathematics Doklady 20 (1979) 191-194.

[35] M. Köppe, Q. Louveaux and R. Weismantel, Intermediate integer programming representations using value disjunctions, Discrete Optimization 5 (2008) 293-313.

[36] H.W. Lenstra, Integer programming with a fixed number of variables, Mathematics of Operations Research 8 (1983) 538-548.

[37] L. Lovász and A. Schrijver, Cones of matrices and set-functions and 0-1 optimization, SIAM Journal of Optimization 1 (1991) 166-190. 
[38] R.R. Meyer, On the Existence of Optimal Solutions to Integer and Mixed Integer Programming Problems, Mathematical Programming 7 (1974) 223-235.

[39] H. Minkowski, Geometrie der Zahlen (Erste Lieferung), Teubner, Leipzig (1896).

[40] G.L. Nemhauser and L.A. Wolsey, A recursive procedure to generate all cuts for 0-1 mixed integer programs, Mathematical Programming 46 (1990) 379-390.

[41] J.H. Owen and S. Mehrotra, On the value of binary expansions for general mixed-integer linear programs, Operations Research 50 (2002) 810-819.

[42] M. Perregaard, A practical implementation of lift-and-project cuts: A computational exploration of lift-and-project cuts with XPRESS-MP, 18th ISMP, Copenhagen (2003).

[43] J.-S. Roy, "Binarize and project" to generate cuts for general mixedinteger programs, Algorithmic Operations Research 2 (2007).

[44] A. Schrijver, On cutting planes, Annals of Discrete Mathematics 9 (1980) 291-296.

[45] A. Schrijver, Theory of Linear and Integer Programming, Wiley, New York (1986).

[46] H. Sherali and W. Adams, A reformulation-linearization technique for solving discrete and continuous nonconvex problems, Kluwer Academic Publishers, Dordrecht (1999).

[47] J.P. Vielma, A constructive characterization of the split closure of a mixed integer linear program, Operations Research Letters 35 (2007) 29-35. 